\title{
Science with the Murchison Widefield Array
}

Judd D. Bowman ${ }^{1,23}$, Iver Cairns ${ }^{2}$, David L. Kaplan ${ }^{3}$, Tara Murphy ${ }^{2,4}$, Divya Oberoi ${ }^{5}$, Lister Staveley-Smith ${ }^{4,6}$, Wayne Arcus ${ }^{7}$, David G. Barnes ${ }^{8}$, Gianni Bernardi ${ }^{9}$, Frank H. Briggs ${ }^{4}, 10$, Shea Brown ${ }^{11}$, John D. Bunton ${ }^{12}$, Adam J. Burgasser ${ }^{13}$, Roger J. Cappallo ${ }^{14}$, Shami Chatterjee ${ }^{15}$, Brian E. Corey ${ }^{14}$, Anthea Coster ${ }^{14}$, Avinash Deshpande ${ }^{16}$, Ludi deSouza ${ }^{2}, 10$, David Emrich ${ }^{7}$, Philip Erickson ${ }^{14}$, Robert F. Goeke ${ }^{17}$, B. M. Gaensler ${ }^{2,4}$, Lincoln J. Greenhill ${ }^{9}$, Lisa Harvey-Smith ${ }^{12}$, Bryna J. Hazelton ${ }^{18}$, David Herne ${ }^{7}$, Jacqueline N. Hewitt ${ }^{17}$, Melanie Johnston-Hollitt ${ }^{19}$, Justin C. Kasper ${ }^{9}$, Barton B. Kincaid ${ }^{14}$, Ronald Koenig ${ }^{12}$, Eric Kratzenberg ${ }^{14}$, Colin J. Lonsdale ${ }^{14}$, Mervyn J. Lynch ${ }^{7}$, Lynn D. Matthews ${ }^{14}$, S. Russell McWhirter ${ }^{14}$, Daniel A. Mitchell ${ }^{4,9,20}$, Miguel F. Morales ${ }^{18}$, Edward H. Morgan $^{17}$, Stephen M. Ord ${ }^{7}$, Joseph Pathikulangara ${ }^{12}$, Thiagaraj Prabu ${ }^{16}$,

Ronald A. Remillard ${ }^{17}$, Timothy Robishaw ${ }^{2,21}$, Alan E. E. Rogers ${ }^{14}$, Anish A. Roshi' ${ }^{22}$, Joseph E. Salah ${ }^{14}$, Robert J. Sault ${ }^{20}$, N. Udaya Shankar ${ }^{16}$, K. S. Srivani ${ }^{16}$, Jamie B. Stevens ${ }^{11}$, Ravi Subrahmanyan ${ }^{4}, 16$, Steven J. Tingay ${ }^{4,7}$, Randall B. Wayth ${ }^{4,7,9}$, Mark Waterson ${ }^{7,10}$, Rachel L. Webster ${ }^{4,20}$, Alan R. Whitney ${ }^{14}$, Andrew J. Williams ${ }^{6}$, Christopher L. Williams ${ }^{17}$ and J. Stuart B. Wyithe $e^{4,20}$

${ }^{1}$ School of Earth and Space Exploration, Arizona State University, Tempe, AZ, USA

${ }^{2}$ Sydney Institute for Astronomy, The University of Sydney, Sydney, Australia

${ }^{3}$ Physics Department, University of Wisconsin-Milwaukee, Milwaukee, WI, USA

${ }^{4}$ ARC Centre of Excellence for All-sky Astrophysics (CAASTRO), Redfern, NSW, Australia

${ }^{5}$ National Centre for Radio Astrophysics, Pune, India

${ }^{6}$ ICRAR - University of Western Australia, Perth, Australia

${ }^{7}$ ICRAR - Curtin University, Perth, Australia

${ }^{8}$ Monash e-Research Centre, Monash University, Melbourne, Australia

${ }^{9}$ Harvard-Smithsonian Center for Astrophysics, Cambridge, MA, USA

${ }^{10}$ Research School of Astronomy and Astrophysics, The Australian National University, Canberra, Australia

${ }^{11}$ Department of Physics and Astronomy, University of Iowa, Iowa City, IA, USA

${ }^{12}$ CSIRO Astronomy and Space Science, Australia

${ }^{13}$ Department of Physics, University of California San Diego, La Jolla, CA, USA

${ }^{14}$ MIT Haystack Observatory, Westford, MA, USA

${ }^{15}$ Department of Astronomy, Cornell University, Ithaca, NY, USA

${ }^{16}$ Raman Research Institute, Bangalore, India

${ }^{17}$ MIT Kavli Institute for Astrophysics and Space Research, Cambridge, MA, USA

${ }^{18}$ Physics Department, University of Washington, Seattle, WA, USA

${ }^{19}$ School of Chemical and Physical Sciences, Victoria University of Wellington, Wellington, New Zealand

${ }^{20}$ School of Physics, The University of Melbourne, Melbourne, Australia

${ }^{21}$ Dominion Radio Astrophysical Observatory, Penticton, BC, Canada

${ }^{22}$ National Radio Astronomy Observatory, Charlottesville, WV, USA

${ }^{23}$ Corresponding author. Email: judd.bowman@asu.edu

(Received September 12, 2012; Accepted February 26, 2013; Online Publication April 16, 2013)

\begin{abstract}
Significant new opportunities for astrophysics and cosmology have been identified at low radio frequencies. The Murchison Widefield Array is the first telescope in the southern hemisphere designed specifically to explore the low-frequency astronomical sky between 80 and $300 \mathrm{MHz}$ with arcminute angular resolution and high survey efficiency. The telescope will enable new advances along four key science themes, including searching for redshifted $21-\mathrm{cm}$ emission from the EoR in the early Universe; Galactic and extragalactic all-sky southern hemisphere surveys; time-domain astrophysics; and solar, heliospheric, and ionospheric science and space weather. The Murchison Widefield Array is located in Western Australia at the site of the planned Square Kilometre Array (SKA) low-band telescope and is the only low-frequency SKA precursor facility. In this paper, we review the performance properties of the Murchison Widefield Array and describe its primary scientific objectives.
\end{abstract}

Keywords: dark ages, reionisation, first stars - instrumentation: interferometers - radio continuum: general - radio lines: general - Sun: general 


\section{INTRODUCTION}

The Murchison Widefield Array $\left(\mathrm{MWA}^{1}\right)$ is a low-frequency radio telescope operating between 80 and $300 \mathrm{MHz}$. It is located at the Murchison Radio-astronomy Observatory (MRO) in Western Australia, the planned site of the future Square Kilometre Array $\left(\mathrm{SKA}^{2}\right.$ ) low-band telescope, and is one of three telescopes designated as a Precursor for the SKA. The MWA has been developed by an international collaboration, including partners from Australia, India, New Zealand, and the United States. It has begun operations in 2013. In this paper, we review the science capabilities of the telescope and the primary science objectives motivating its design and operation. Details of the technical design and specifications of the MWA as built are presented in a companion article (Tingay et al. 2013), which supersedes the earlier Lonsdale et al. (2009) description of plans for the instrument.

The four key science themes driving the MWA are (1) searching for redshifted $21-\mathrm{cm}$ emission from the epoch of reionisation (EoR) in the early Universe; (2) Galactic and extragalactic surveys; (3) time-domain astrophysics; and (4) solar, heliospheric, and ionospheric science and space weather. We describe the plans and objectives for each of the key science themes in Sections 2-5.

The frequency range targeted by the MWA is one of the more difficult portions of the spectrum for ground-based radio telescopes due to strong distortions of incoming signals by the Earth's ionosphere and also due to congested terrestrial communications, including FM radio, TV stations, aircraft navigation, and satellite communications. Hence, the low-frequency radio sky remains one of the least-explored domains in astronomy. The MRO site mitigates many issues associated with radio interference for the telescope, but processing of MWA observations requires particular care to correct for the ionospheric distortions.

The unifying design theme for the MWA is that of high survey efficiency. Many of the science goals require large areas of the sky to be surveyed and the EoR objective further requires very high surface brightness sensitivity. It was recognised early in the development of the MWA that arcminute angular resolution is a convenient balance between the opposing tensions of maximizing scientific returns and minimizing technical complexity. Arcminute angular scales are sufficient to probe typical ionised bubbles during the EoR, study diffuse and polarised emission structures in the Galaxy, reduce extragalactic source confusion, image bursts in the solar corona near the Sun's surface, and localise transient events with sufficient precision to allow follow-on searches at other wavelengths. In order to achieve arcminute resolution, the longest baseline of the MWA is $3 \mathrm{~km}$. This distance is within the scale size of a typical isoplanatic patch in the ionosphere, allowing simplifying assumptions to be employed for widefield ionospheric calibration and keeping real-time imaging computations within reach of modest computer clusters.

\footnotetext{
${ }^{1} \mathrm{http}: / /$ mwatelescope.org

${ }^{2}$ http://skatelescope.org
}

Table 1 System parameters for the MWA.

\begin{tabular}{ll}
\hline \hline Parameter & ${ }^{a}$ Value \\
\hline Number of tiles & 128 \\
Tile area $\left(\mathrm{m}^{2}\right)$ & 21.5 \\
Total collecting area $\left(\mathrm{m}^{2}\right)$ & 2752 \\
Receiver temperature (K) & 50 \\
Typical sky temperature (K) & $350^{b}$ \\
Field of view (deg $\left.{ }^{2}\right)$ & 610 \\
Angular resolution (arcmin) & 2 \\
Frequency range (MHz) & $80-300$ \\
Instantaneous bandwidth (MHz) & 30.72 \\
Spectral resolution (MHz) & 0.04 \\
Temporal resolution (s) & 0.5 \\
Minimum baseline (m) & 7.7 \\
Maximum baseline (m) & 2864 \\
Estimated confusion limit (mJy) & 10 \\
\hline \hline
\end{tabular}

${ }^{a}$ For frequency-dependent parameters, listed values are given at $150 \mathrm{MHz}$. ${ }^{b}$ Sky temperature varies considerably with Galactic latitude. Here, we use typical values from Nijboer, Pandey-Pommier, \& de Bruyn (2009) and Rogers \& Bowman (2008).

The system properties of the MWA are described in detail by Tingay et al. (2013). We summarise them here in Table 1. The time needed to reach a point source sensitivity of $\sigma_{\mathrm{s}}$ for the MWA is given by

$$
t=\left(\frac{2 k_{\mathrm{B}} T}{A_{\text {eff }} N \epsilon_{\mathrm{c}}}\right)^{2} \frac{1}{\sigma_{\mathrm{s}}^{2} B n_{\mathrm{p}}},
$$

where $k_{\mathrm{B}}$ is the Boltzmann constant, $T=T_{\text {sky }}+T_{\mathrm{rcv}}$ is the system temperature, $A_{\text {eff }}$ is the effective area of each antenna tile, $N$ is the number of antenna tiles, $\varepsilon_{\mathrm{c}}$ is the correlator efficiency (assumed to be unity), $B$ is the instantaneous bandwidth, and $n_{\mathrm{p}}$ is the number of polarisations. For continuum imaging at $150 \mathrm{MHz}$, this reduces to $t \approx 8 \times 10^{4} /\left(B \sigma_{s}^{2}\right)$ s for $B$ in units of $\mathrm{MHz}$ and $\sigma_{s}$ in units of mJy. The intrinsic source confusion limit for the MWA angular resolution of 2 arcmin is estimated to be $\sim 10 \mathrm{mJy}$ at $150 \mathrm{MHz}$ (Condon 1974; Windhorst et al. 1985; Condon et al. 1998; Subrahmanyan \& Ekers 2002). Hence, the MWA will reach the confusion limit in $\sim 25 \mathrm{~s}$ for continuum imaging or within several hours in a single 40-kHz spectral channel.

The surface brightness sensitivity of the MWA varies significantly with angular scale. The MWA antenna layout emphasises a dense, central core in order to achieve a high surface brightness sensitivity on degree scales for EoR science. For a typical EoR spectral resolution of $1 \mathrm{MHz}$, the brightness temperature uncertainty is less than $150 \mathrm{mK}$ after $1 \mathrm{~h}$ of integration on degree scales, whereas it is approximately $3 \mathrm{~K}$ on arcminute scales.

In the remainder of the paper, we discuss the four key science themes. Section 2 describes the EoR science. In Section 3 , broad categories of radio astronomy science are discussed within the context of sky surveys. Section 4 describes the time-domain astronomy that will be possible with the MWA and Section 5 presents the primary solar, heliospheric, and ionospheric science goals. We conclude in Section 6. 


\section{EPOCH OF REIONISATION}

One of the most significant events in the history of the intergalactic medium (IGM) was the reionisation of hydrogen. The EoR marks the time when the small fraction of matter within galaxies significantly affected their surroundings (and hence future generations of galaxies), ionising the intergalactic gas and heating it from tens of Kelvin to $\sim 10^{4} \mathrm{~K}$.

The MWA has been designed with the goal of detecting $21-\mathrm{cm}$ emission from the reionisation epoch. It is one of three new radio arrays specifically targeting this science objective, along with PAPER ${ }^{3}$ and LOFAR. ${ }^{4}$ The 21 -cm hyperfine transition line of neutral hydrogen is anticipated to provide an ideal probe of reionisation. Its weak oscillator strength (in contrast to the Ly $\alpha$ line) allows $21-\mathrm{cm}$ photons to propagate from fully neutral environments at high redshifts without appreciable absorption. This property makes it possible to directly observe the neutral hydrogen gas before and during reionisation.

Unlike the continuum emission of the cosmic microwave background $(\mathrm{CMB}), 21-\mathrm{cm}$ emission contains information in the frequency (or redshift) dimension in addition to in angle, enabling the study of the full history of reionisation. Redshifted 21-cm emission from neutral hydrogen gas in the IGM should appear as a faint, diffuse background in radio frequencies below $v \leqslant 200 \mathrm{MHz}$ for redshifts above $z>6$, where $v=1420 /(1+z)$ MHz. At these redshifts, the expected unpolarised differential brightness temperature, $\delta T_{21}$, of the redshifted $21-\mathrm{cm}$ line relative to the CMB is readily calculable from basic principles (see Furlanetto, Oh, $\&$ Briggs 2006, for a complete review) and is given by

$$
\begin{aligned}
\delta T_{21}(\boldsymbol{\theta}, z) \approx & 25 x_{\mathrm{HI}}(1+\delta)\left(\frac{1+z}{10}\right)^{1 / 2} \\
& \times\left[1-\frac{T_{\gamma}(z)}{T_{\mathrm{S}}}\right]\left[\frac{H(z) /(1+z)}{\mathrm{d} v_{\|} / \mathrm{dr}_{\|}}\right] \mathrm{mK},
\end{aligned}
$$

where $x_{\mathrm{HI}}$ is the neutral fraction of hydrogen, $\delta$ is the local overdensity in baryons, $T_{\gamma}(z)=2.73(1+z) \mathrm{K}$ is the temperature of $\mathrm{CMB}$ at the redshift of interest, $T_{\mathrm{S}}$ is the spin temperature that describes the relative population of the ground and excited states of the $21-\mathrm{cm}$ transition, $H(z)$ is the Hubble constant, and $\mathrm{d} v_{\|} / \mathrm{dr}_{\|}$is the velocity gradient along the line of sight.

All of these factors contain unique astrophysical information. The dependence on $\delta$ traces the development of the cosmic web (Scott \& Rees 1990), while the velocity factor creates line-of-sight distortions that can be used to separate different components of the signal (Barkana \& Loeb 2005). The other two factors depend on the ambient radiation fields in the early Universe. The local neutral fraction, $x_{\mathrm{HI}}$, is determined by the ionising radiation emitted by galaxies and possibly quasars. Theoretical studies suggest that the distribution

\footnotetext{
${ }^{3}$ http://eor.berkeley.edu

${ }^{4}$ http://www.lofar.org
}

of neutral gas during reionisation is very inhomogeneous (e.g. McQuinn et al. 2007). Finally, the 21-cm spin temperature, $T_{\mathrm{S}}$, is determined primarily by three mechanisms that affect the hyperfine level populations: the ambient radiation at $21 \mathrm{~cm}$ due to the $\mathrm{CMB}$ with $T_{\gamma}$, the ultraviolet background that tends to drive $T_{\mathrm{S}}$ to the gas temperature (Wouthuysen 1952; Field 1958), and heating from the cosmological X-ray background (Chen \& Miralda-Escudé 2004). By the time the Universe is a few per cent ionised, most calculations predict that $T_{\mathrm{S}} \gg T_{\gamma}$, such that fluctuations in $T_{\mathrm{S}}$ are not important (e.g. Furlanetto et al. 2006).

Figure 1 illustrates how the factors in Equation (2) combine to create the observable $21-\mathrm{cm}$ signal. The panels in this figure are from the simulations of McQuinn et al. (2007) and are $100 \mathrm{cMpc}$ across or $\sim 0.5^{\circ}$ in angle (a small fraction of the $\sim 20^{\circ}$ MWA field of view). The left panel of Figure 1 shows the expected signal just before reionisation begins, when the fluctuations are dominated by local perturbations in the matter density. In the middle panel, reionisation is underway $\left(x_{\mathrm{HI}} \approx 0.5\right)$, and the large-scale voids in the map signify ionised regions. After reionisation has largely finished (right panel), only localised pockets of neutral hydrogen remain.

The MWA will attempt to probe structures in the redshifted 21-cm background in order to reveal the astrophysics of reionisation. The MWA is expected to be sensitive to the power spectrum of the $21-\mathrm{cm}$ signal over the redshift range $6<z \lesssim 10$ (Section 2.1). Its observations have the potential to characterise the nature of the sources that are responsible for ionising the IGM, chart the evolution of the global neutral fraction, and probe the nature of quasar emissions by constraining the properties of their ionised proximity zones.

\subsection{1-cm power spectrum}

Initial MWA observations aimed at detecting 21-cm emission will focus on two target regions at relatively high Galactic latitudes (see Figure 2). Over the course of one year, approximately $1000 \mathrm{~h}$ of optimal nighttime observations will be divided between these two regions. The signal-to-noise ratio $(\mathrm{S} / \mathrm{N})$ in each pixel will be $\lesssim 1$, with the highest $\mathrm{S} / \mathrm{N}$ on the largest angular modes. Hence, rather than imaging the $21-\mathrm{cm}$ brightness temperature in the target fields, reionisation science with the MWA will focus on statistical measures of the 21-cm emission. The number of independent measurements in a raw, multi-frequency MWA data cube will be $\sim 10^{8}$. These samples record largely Galactic synchrotron and freefree emission $(\gtrsim 200 \mathrm{~K})$ and extragalactic continuum sources $(\sim 50 \mathrm{~K})$, with only a small contribution from the redshifted 21-cm signal $(\sim 25 \mathrm{mK})$. The primary approach planned for the MWA is to calculate the power spectrum of spatial fluctuations of the $21-\mathrm{cm}$ signal.

The three-dimensional power spectrum of redshifted 21$\mathrm{cm}$ emission is the square of the Fourier transform of $\delta T_{21}(2)$. It has a large spherically symmetric component due to the isotropy of space, but has slightly more power for modes that point in the line-of-sight direction that results from 

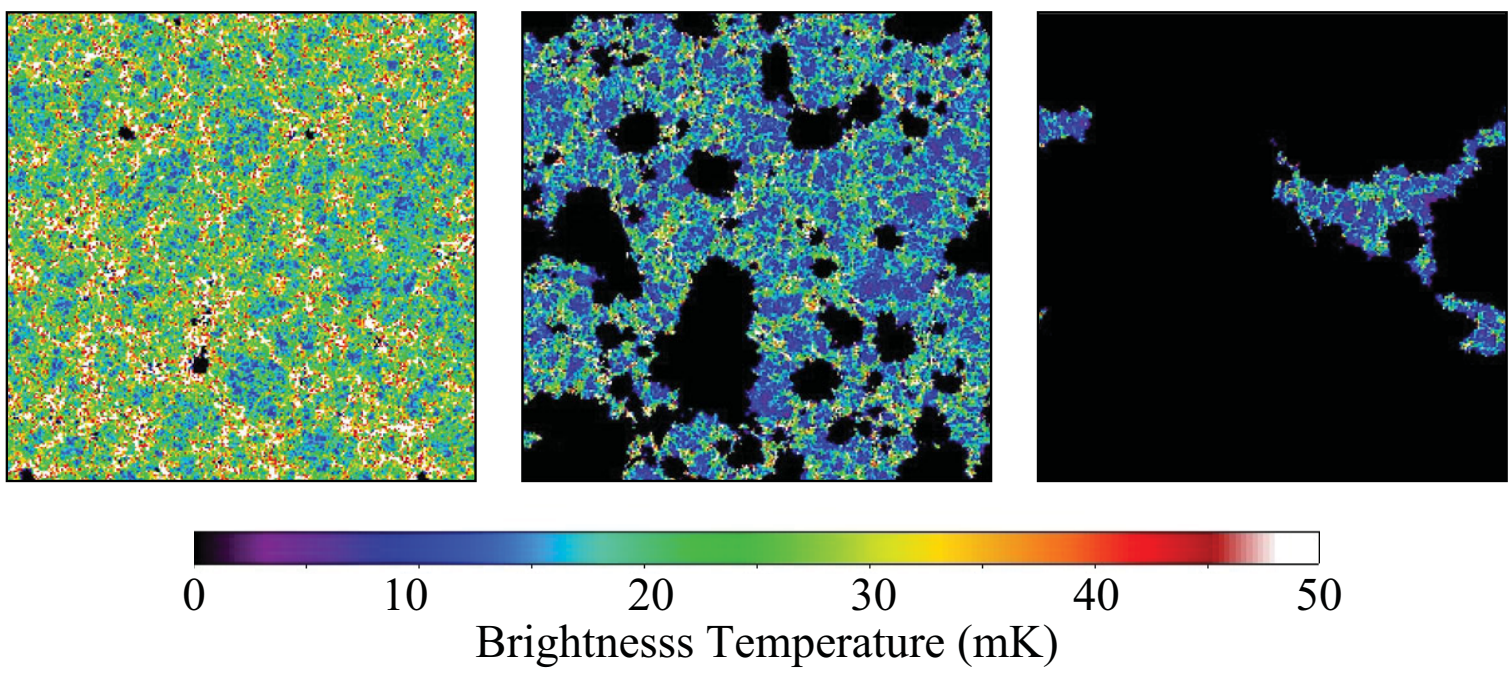

Figure 1. Simulated 21-cm brightness temperature maps from simulation S1 by McQuinn et al. (2007) at three epochs calculated under the assumption that $T_{\mathrm{S}} \gg T_{\gamma}$. The left-hand panel is an almost fully neutral IGM (before reionisation at $z=12$ ) such that the 21-cm emission traces the matter density. The middle panel is during reionisation $\left(x_{\mathrm{HI}} \approx 0.5\right.$, which occurs at $z=8$ in this simulation): the dark regions represent ionised regions around galaxies. Lastly, the right-hand panel shows the residual emission near the end of reionisation $(z=6)$. Each panel is $100 \mathrm{cMpc}$ across and subtends $0.5^{\circ}$ (a small fraction of the MWA field of view).

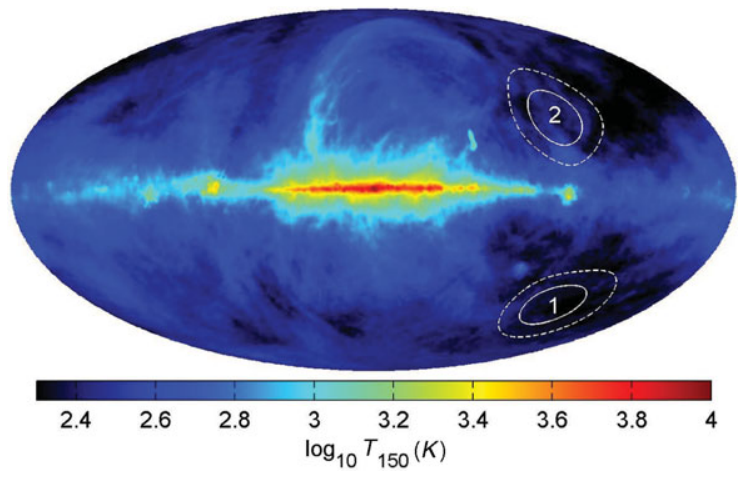

Figure 2. Projected all-sky brightness temperature map of de OliveiraCosta et al. (2008) at $150 \mathrm{MHz}$. The primary (1) and secondary (2) target regions for EoR observations are centred at $\alpha, \delta=60^{\circ},-30^{\circ}\left(\ell, b=228^{\circ}\right.$, $\left.-49^{\circ}\right)$ and $\alpha, \delta=155^{\circ},-10^{\circ}\left(\ell, b=253^{\circ},+38^{\circ}\right)$, respectively. The solid white curves indicate the $50 \%$ response power contour of the primary antenna tile beams for reference and the dashed lines show the $10 \%$ power contour.

peculiar velocities. The predicted components in the full three-dimensional power spectrum can be decomposed into terms with different powers of $\mu \equiv \hat{k} \cdot \hat{n}$, where $\mu$ is the cosine of the angle between the line of sight and the wavevector (Kaiser 1987; Barkana \& Loeb 2005; McQuinn et al. 2006). Thus,

$$
P_{21}(\boldsymbol{k})=P_{\mu^{0}}(k)+\mu^{2} P_{\mu^{2}}(k)+\mu^{4} P_{\mu^{4}}(k) .
$$

During reionisation, the $P_{\mu^{0}}$ term typically dominates, and the most important contribution to $P_{\mu^{0}}$ is the fluctuations in the neutral fraction (McQuinn et al. 2006).

Averaging $P_{21}(\boldsymbol{k})$ over angle to yield $P_{21}(k)$ loses some statistical information, but at the sensitivity of the MWA this loss is minor during reionisation. With one year of the planned observing programme, the MWA can constrain $P_{21}(k)$ at a given redshift and across a range of scales spanning $0.01 \lesssim k \lesssim 1$ $\mathrm{cMpc}^{-1}$ with total $\mathrm{S} / \mathrm{N} \approx 14$ (Morales \& Hewitt 2004; Bowman, Morales, \& Hewitt 2006; McQuinn et al. 2006; Beardsley et al. 2013). The sensitivity of the MWA to the spherically averaged power spectrum is shown in Figure 3.

The properties of the power spectrum, $P_{21}(k)$, during reionisation are dominated by the characteristics of the ionised bubbles (Figure 1, middle panel) that are determined by the ionising radiation produced by the first galaxies and quasars. Figure 3 illustrates how $\Delta_{21} \equiv\left(k^{3} P_{21}(k) / 2 \pi^{2}\right)^{1 / 2}$ contains information about the reionisation process. It plots $\Delta_{21}$ for the fiducial model of Lidz et al. (2008) at several neutral fractions. The shape of the power spectrum evolves considerably as the hydrogen in the IGM becomes ionised, with power shifting from small scales initially, to larger scales later when large ionised bubbles have formed. Eventually, the amplitude decreases as hydrogen reionisation completes.

The sensitivity forecasts shown in Figure 3 utilise both the primary and secondary target fields in combination, assuming that each field has been observed over an 8$\mathrm{MHz}$ bandwidth (corresponding to $\Delta z \approx 0.5$ ). The full 32$\mathrm{MHz}$ instantaneous bandwidth of the MWA will yield four such measurements spanning a contiguous redshift range of $\Delta z \approx 2$. Initial observations will focus on the lower redshifts $(6<z<8)$, where the sensitivity of the instrument is highest due to the lower sky temperature. Observations at low redshifts will crucially demonstrate whether the foreground subtraction is successful, since the quality of the foreground subtraction will be readily apparent at $z<6$, where no 21$\mathrm{cm}$ signal should be detectable by the MWA since $x_{\mathrm{HI}} \lesssim 2 \%$ after reionisation (Wyithe \& Loeb 2008). 


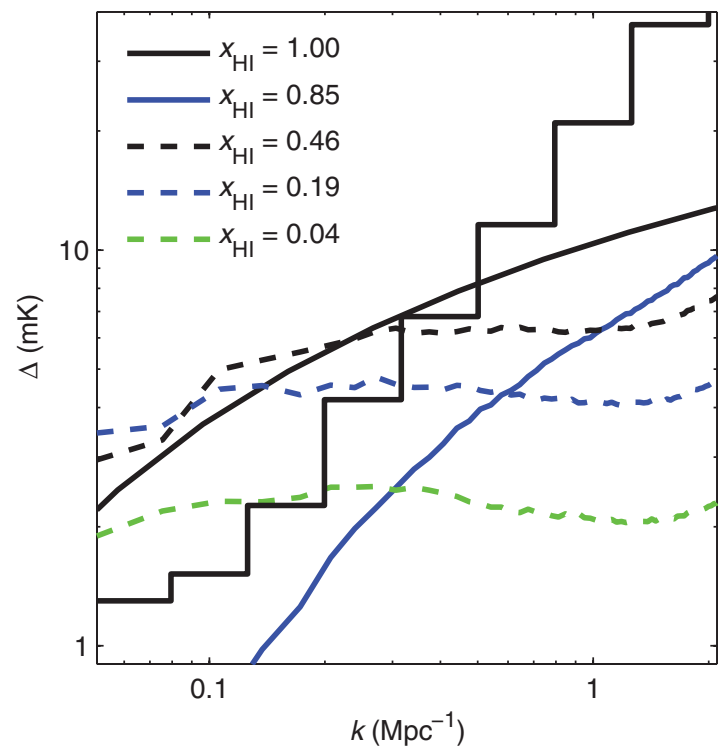

Figure 3. MWA thermal uncertainty at $z=8$ with simulated $21-\mathrm{cm}$ power spectra. The black stepped line is the $1 \sigma$ thermal uncertainty modelled for a combined observation of the primary and secondary EoR fields with a total of $1600 \mathrm{~h}$ (Beardsley et al. 2013). The 21-cm curves are calculated using the fiducial simulation described by Lidz et al. (2008). The power spectrum is very steep at the beginning of reionisation $\left(x_{\mathrm{HI}} \gtrsim 0.8\right)$ and falls rapidly at the scales probed by the MWA. Large-scale power returns as large ionised regions form by $x_{\mathrm{HI}} \approx 0.5$ and then it falls again with decreasing neutral fraction. The MWA should be able to detect the power spectrum at $z=8$ for any appreciable neutral fraction outside of the range $0.95 \gtrsim x_{\mathrm{HI}} \gtrsim 0.8$.

\subsubsection{Sources of reionisation}

The 21-cm sky contains a wealth of information about the properties of the ionising sources because they strongly affect the topology of the EoR. Reionisation scenarios dominated by stars in galaxies produce well-defined ionised regions, while 'miniquasars' (small accreting black holes) yield more diffuse features (Zaroubi \& Silk 2005) and reionisation processes involving decaying/annihilating dark matter particles would be very inhomogeneous (Belikov \& Hooper 2009). The 21-cm observations could even distinguish between different stellar reionisation scenarios (Lidz et al. 2008). For example, more massive galaxies produce larger ionised regions (Furlanetto, Zaldarriaga, \& Hernquist 2004; McQuinn et al. 2007). If it is able to constrain the shape and evolution of the power spectrum, the MWA will reveal the nature of some of the first cosmological objects.

\subsubsection{The neutral fraction}

Existing observations have provided tantalising hints about reionisation, but even more unanswered questions. CMB observations find that the average redshift of reionisation is $z=$ $10 \pm 2$ (Komatsu et al. 2009), and quasar absorption spectra imply that the Universe was totally ionised by $z=6$, one billion years after the big bang. In addition, several studies have interpreted the large saturated regions at $z>6$ in these absorption spectra as evidence for the end of reionisation (e.g. Fan et al. 2006), but this conclusion is disputed (Becker, Rauch, \& Sargent 2007; Mesinger 2010).

Detection of the $21-\mathrm{cm}$ power spectrum will yield measures of $\bar{x}_{\mathrm{Hi}}(z)$ through predictable features that depend on the neutral fraction. As evident in Figure 3, the intensity of $10 \mathrm{cMpc}$ scale fluctuations in $21-\mathrm{cm}$ brightness temperature briefly fades when reionisation begins and the first galaxies ionise the overdense regions within a couple of $\mathrm{cMpc}$ of them. Next, the ionised regions surrounding individual galaxies overlap, creating $\sim 10 \mathrm{cMpc}$ ionised regions and an increase in power on these scales. Finally, all of the fluctuations fade as these bubbles overlap, swallowing the neutral hydrogen that remains. Each of these trends can be used to disentangle $\bar{x}_{\mathrm{Hi}}(z)$.

For many possible sources that have been considered in the literature (such as galaxies, quasars, and mini-halos), the amplitude of the power spectrum peaks at $\bar{x}_{\mathrm{Hi}} \approx 50 \%$. The MWA could probe the global ionisation history by tracking the evolution of this amplitude and shape of the $21-\mathrm{cm}$ power spectrum. Using these features, the volume-filling factor of neutral hydrogen (comparable to the mass-averaged neutral fraction) can be constrained after approximately two years of observations to between $0.4<x_{\mathrm{HI}}<0.75$ at $2 \sigma$ significance for the redshift where the power spectrum achieves its maximum amplitude (Lidz et al. 2008). While unlikely, it is possible that the IGM is highly ionised over the range of redshifts measured by the MWA. Lidz et al. (2008) found that a null detection with the MWA at $z \leq 8$ would be able to constrain the neutral fraction to $\$ 4 \%$.

Observations of individual quasar $\mathrm{H}$ II regions offer an additional probe of the global neutral hydrogen fraction since the contrast between the ionised $\mathrm{H}$ II region and neutral IGM can provide a measurement of the neutral fraction at the redshift of the quasar. Geil et al. (2008) have found that the shape of the quasar $\mathrm{H}$ II regions can be recovered from foreground subtracted MWA maps by using the entire data cube to help determine the contrast between the external IGM and ionised interior.

\subsection{Quasar H II regions}

The detection of large quasar-generated H II regions in redshifted 21-cm emission late in the EoR may be possible with the MWA (Wyithe, Loeb, \& Barnes 2005; Wyithe 2008). Geil et al. (2008) have modelled the observation of quasar $\mathrm{H}$ II regions in a partially ionised IGM. Figure 4 shows a simulated example of a recovered quasar $\mathrm{H}$ II region with the MWA after approximately one observing season. The recovery is possible only for quasars with positions known a priori. Although the EoR fields will not overlap the Sloan Digital Sky Survey (SDSS) fields, where the existing known $z \approx 6$ quasars reside, the contemporaneous SkyMapper optical survey (Keller et al. 2007) should find around one quasar at $z>6.25$, and around 5 quasars at $z>6$ per MWA field.

Like all $21-\mathrm{cm}$ signatures of the reionisation era, the detection of a quasar $\mathrm{H}$ II region will need to overcome the 

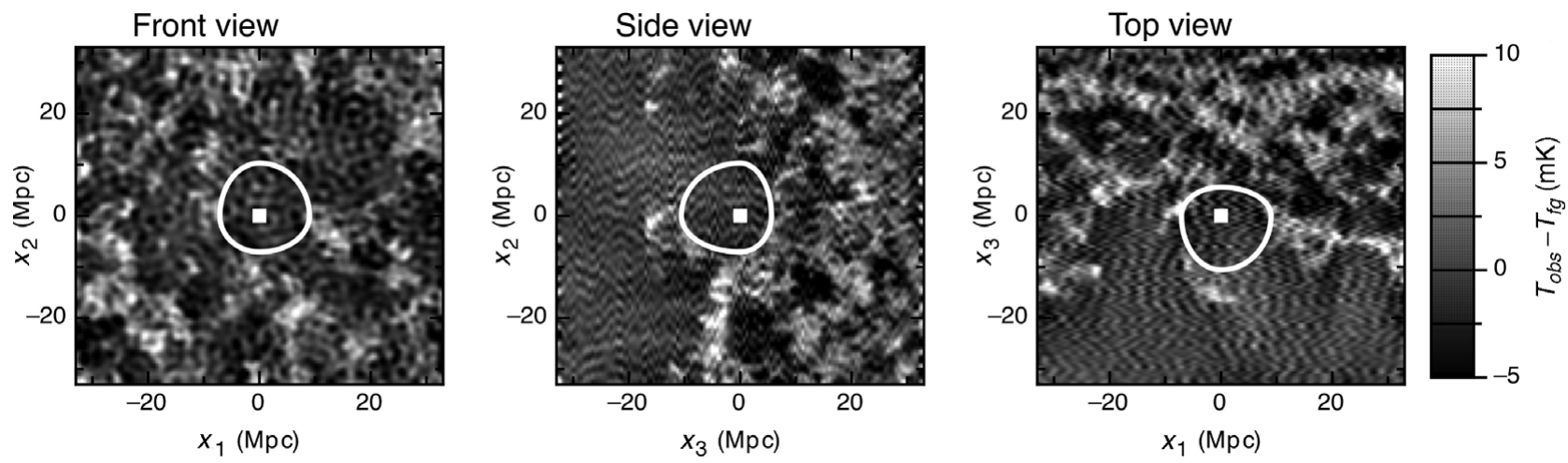

Figure 4. Simulated recovery of a quasar H II region in an evolving IGM, as observed by an MWA-like instrument (Geil et al. 2008). Foregrounds were simulated and removed for the maps shown. The data cube is only a small part of a full MWA field and spans $\sim 3.3^{\circ}$ on a side and $33 \mathrm{MHz}$ deep. The three panels show different slices through the centre of the box when viewed from the front, side, and top. Each slice is $6 \mathrm{cMpc}$ thick, which corresponds to $\sim 3 \mathrm{MHz}$ along the $x_{3}$-axis. The extracted shape of the $\mathrm{H}$ II region is shown, based on a nine-parameter model. The quasar was assumed to have a lifetime of $4 \times 10^{7} \mathrm{yr}$ centred on $z=6.65$ and to have produced an $\mathrm{H}$ II region with a radius of $34 \mathrm{cMpc}$ at that redshift. The mass-averaged IGM neutral fraction at the redshift of the quasar is $\sim 15 \%$.

difficulties associated with the removal of bright Galactic and extragalactic foregrounds. Geil et al. (2008) find that the primary effect of continuum foreground removal is to reduce contrast in the image. In particular, contributions to the 21$\mathrm{cm}$ intensity fluctuations that have a scale length comparable to the frequency bandpass of the observation are removed by foreground subtraction. This loss of contrast, however, does not severely affect the ability of $21-\mathrm{cm}$ observations to measure the size and shape of the $\mathrm{H}$ II region. To demonstrate this quantitatively, Geil et al. (2008) modelled the H II region shape as a spheroid described by six parameters and showed that the shape recovered following foreground removal agrees well with the shape derived directly from fitting using the input $\mathrm{H}$ II region model.

Using the recovered best-fit shape of the $\mathrm{H}$ II region, the global neutral fraction of hydrogen in the IGM can be measured directly from the contrast in intensity between regions that are within and beyond the $\mathrm{H}$ II region. However, since foreground removal lowers the observed contrast between the $\mathrm{H}$ II region and the IGM, such a measurement of the neutral fraction requires a correction factor. The value of this correction factor depends on the reionisation history and the polynomial used for foreground subtraction and can be accounted for using astrophysical models.

\subsection{Non-Gaussian statistics}

During the reionisation epoch, fluctuations in the $21-\mathrm{cm}$ emission are expected to be highly non-Gaussian due to galaxy bias and the patchy structure imparted by the ionised bubbles in the IGM. The power spectrum (or two-point correlation function), therefore, is not a sufficient descriptor of the properties of the field, and additional statistical measures can provide complementary information. Several extensions to the power spectrum have been proposed in this context, including the 21-cm galaxy cross spectrum (Furlanetto et al. 2006; Wyithe, Loeb, \& Schmidt 2007; Lidz et al.
2009), directly measuring the one-point probability distribution function (PDF) of the 21-cm brightness temperature distribution as a function of smoothing scale (Mellema et al. 2006; Ichikawa et al. 2010; Petrovic \& Oh 2011) or its higher moments (Wyithe \& Morales 2007), the three-point correlation function (bispectrum), matched filters (Datta et al. 2008), the threshold clustering function, and other derived metrics (Barkana \& Loeb 2008).

\subsection{Foreground subtraction}

The ability of the MWA to achieve its reionisation science is entirely contingent on the successful separation of the $21-\mathrm{cm}$ signal from astrophysical foregrounds. Hence, developing and demonstrating the techniques required to subtract foregrounds is a critical research objective.

\subsubsection{Reference pipeline}

The baseline foreground subtraction strategy is a multi-stage process consisting of the following principal stages:

1. Bright source subtraction. The first step in foreground subtraction is the removal of bright points sources. This is necessary because the sidelobes of these sources mask the 21-cm signal. Recent theoretical studies have indicated that bright point source subtraction must be complete for sources with flux greater than $S \gtrsim 100 \mathrm{mJy}$ in order to allow for successful subsequent Galactic continuum foreground subtraction (Liu, Tegmark, \& Zaldarriaga 2009). The brightest sources in the field will be 'peeled' (self-calibrate and subtract) from the raw visibility measurements (Mitchell et al. 2008). Efficient techniques to remove point sources from gridded and integrated maps are also under investigation (Pindor et al. 2011; Bernardi et al. 2011).

2. Diffuse continuum subtraction. A variety of mechanisms contribute to diffuse continuum emission in the low-frequency radio sky including Galactic synchrotron 


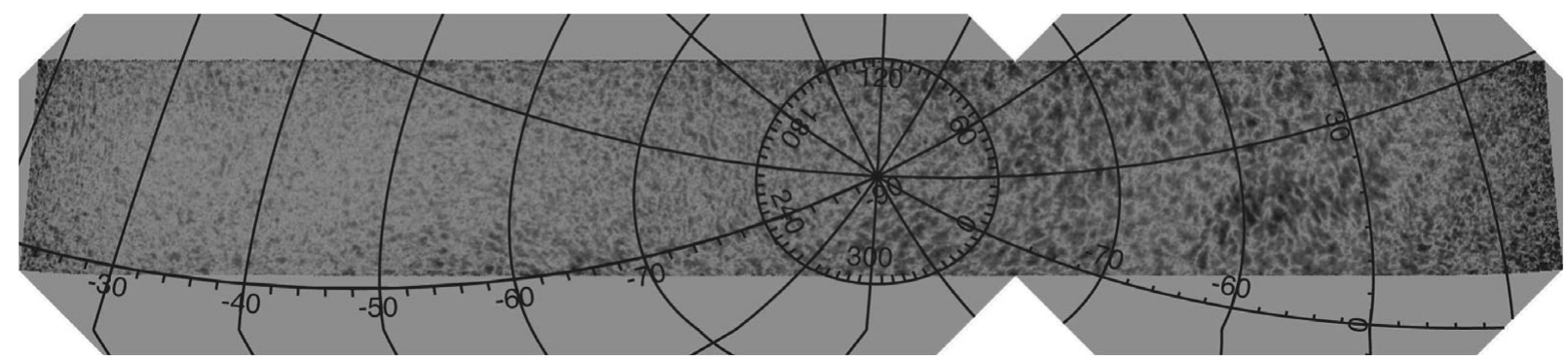

Figure 5. Polarised intensity at Faraday depth zero at $189 \mathrm{MHz}$ from the MWA development system (Bernardi et al. 2013). The field spans $2400 \mathrm{deg}^{2}$ with 15-arcmin angular resolution and is roughly centred on the southern Galactic pole, extending to within $20^{\circ}$ of the Galactic plane. The radial lines are Galactic latitude and the circular arcs indicate Galactic longitude. Flux density is plotted on a linear colour scale from 0 to $0.2 \mathrm{Jy}_{\text {beam }}{ }^{-1} \mathrm{RMSF}^{-1}$ (white to black), where RMSF is the RM spread function. Polarised maps from the MWA showing similar complex structures and discontinuities that often have no counterparts in total intensity will be used to determine the properties of both ordered and turbulent magnetic fields in the Milky Way's ISM (see Figure 6).

emission, Galactic free-free emission, extragalactic freefree emission, and a sea of faint extragalactic point sources that are too numerous to identify individually in maps and are, thus, said to be 'confused'. All of these foregrounds are significantly stronger than the redshifted $21-\mathrm{cm}$ signal and have considerable angular structure on the sky (see Figure 2; Santos, Cooray, \& Knox 2005, their Figure 5). But the emission mechanisms of these foregrounds produce smooth spectra that follow power-law profiles, making them possible to separate from the redshifted $21-\mathrm{cm}$ emission by their long spectral coherence scales compared with the small, $\sim 1-\mathrm{MHz}$ spectral coherence of the reionisation signal. A variety of techniques have been proposed to accomplish this separation including polynomial fits along the spectral axis of each pixel in an image map or its uv-map conjugate, Minkowsky functionals, and non-parametric techniques. The baseline approach planned for the MWA is to fit and subtract a thirdorder polynomial from each pixel in the uv-domain representations of the bright source cleaned maps. This technique has been demonstrated for the MWA in the limit of perfect instrumental calibration (Liu et al. 2009a; Bowman, Morales, \& Hewitt 2009; Liu et al. 2009b).

3. Polarised leakage subtraction. In addition to the foreground issues discussed thus far, gain and phase calibration errors and non-ideal feeds conspire to contaminate all Stokes parameters further. This leakage leads to some portion of the complex linear polarisation signal finding its way into the Stokes I intensity maps that will be used for the redshifted 21$\mathrm{cm}$ measurements. By using rotation measure (RM) synthesis (Brentjens \& de Bruyn 2005; see also Section 3.3) to exploit the Fourier relationship between the polarised signal and the Faraday dispersion function, it is possible to identify components of the polarised signal with specific Faraday depth. The three-dimensional Stokes $I$ data set is first re-sampled along the frequency axis into the natural $\lambda^{2}$-basis for Faraday rotated emission. A one-dimensional Fourier transform is then applied along each line of sight. Any feature observed with a non-zero Faraday depth in the three-dimensional RM synthesis map will, by definition, be polarised contamination. By applying an inversion process together with knowledge of the instrument's specific leakage behaviour, this contamination can be subtracted (Geil, Gaensler, \& Wyithe 2011).

4. Statistical template fitting and power spectrum estimation. Many classes of errors and residuals remaining in the maps after the foreground cleaning stages described above can be modelled given sufficient knowledge of the instrumental response (Morales, Bowman, \& Hewitt 2006; Bowman et al. 2009; Datta, Bowman, \& Carilli 2010; Morales et al. 2012). The statistical properties of these residuals can be exploited to provide an additional layer of foreground removal by fitting templates of the residual statistical structures during the final redshifted $21-\mathrm{cm}$ power spectrum parameter estimation. This technique is now commonly employed in CMB power spectrum analysis to account for the angular power of faint point sources and the effects of gravitational lensing.

\subsubsection{Unified parameter estimation}

The stages of foreground subtraction outlined above can be performed discretely, resulting in a series of output products, the last of which is used as the input into power spectrum estimation and other statistical tests to extract information from the redshifted $21-\mathrm{cm}$ signal. Alternatively, they can be performed in a unified matrix framework that simultaneously fits models for the foreground contributions and the $21-\mathrm{cm}$ statistical signal (Liu \& Tegmark 2011; Tegmark 1997). The latter has the advantage of producing an optimal result that minimizes both bias (from thermal noise and foreground contamination) and correlations between parameters, and yields the smallest possible error bars, but at the expense of a large computational overhead. Advances in processing power and new efforts at sparse matrix reduction have begun to enable the trial application of unified parameter estimation in simulations and preliminary data, and it is expected this approach will ultimately be the preferred technique for data analysis.

\section{GALACTIC AND EXTRAGALACTIC SCIENCE}

The MWA offers several exciting science opportunities under the collective banner 'Galactic and Extragalactic' 
science. The major input into this science will come from a deep all-sky southern hemisphere survey over the MWA frequency range with full polarimetry and spectral resolution. This survey is a critical undertaking as it will also be used by the other science programmes as a basis for an all-sky foreground model and the identification of calibrators. The data will also be combined with other new surveys, including GALFACTS and POSSUM (1.4 GHz), S-PASS (2.4 GHz), and Planck (27 GHz to $1 \mathrm{THz}$ ) surveys.

The following sections describe a selection of Galactic and extragalactic experiments for which the MWA is well suited. These projects capitalise on three advantages of the MWA's low observing frequencies: (1) non-thermal sources will be very bright; (2) Faraday rotation (FR) and depolarisation will be very strong; and (3) along some sightlines free-free absorption will be significant.

\subsection{The cosmic web}

Theory suggests that the infall of baryons into the cosmic web results in high Mach number intergalactic shocks (e.g. Cen \& Ostriker 1999; Ryu et al. 2003). When combined with seed magnetic fields, efficient cosmic ray acceleration is possible, resulting in steep-spectrum synchrotron emission (Keshet, Waxman, \& Loeb 2004; Pfrommer et al. 2006; Pfrommer, Enßlin, \& Springel 2008; Skillman et al. 2011; Araya-Melo et al. 2012). This may be a ubiquitous component of the extragalactic radio sky (Keshet et al. 2004), and recent simulations suggest that the majority of the luminous radio shocks can be found in the outskirts of massive galaxy clusters (ArayaMelo et al. 2012). The MWA's centrally condensed antenna configuration provides the theoretical sensitivity required to make the maps of this relativistic cosmic web. However, the accurate subtraction of discrete sources requires long baselines and high image fidelity (Brown 2011). This may limit direct to the densest portions of the web, though statistical detection may be possible (Keshet et al. 2004; Brown, Farnsworth, \& Rudnick 2010).

\subsection{Radio relics and clusters}

The MWA is also well suited for studying the diffuse, steepspectrum synchrotron emission from clusters of galaxies. These objects, which include the centrally located 'radio halos' and peripheral 'radio relics' (Kempner et al. 2004), are probes of relativistic electrons and large-scale magnetic fields in the intra-cluster medium (ICM; Ferrari et al. 2008; Rudnick et al. 2009). Possible mechanisms for accelerating the cosmic-ray electrons responsible for this emission are turbulent re-acceleration following a cluster merger (Brunetti et al. 2001; Petrosian 2001) and hadronic collisions between cosmic-ray and thermal protons (Dennison 1980; Blasi \& Colafrancesco 1999). Low-frequency observations of clusters can probe these different models (Cassano et al. 2010, 2012). The MWA's surface-brightness sensitivity will enable it to detect very steep-spectrum halos and relics. Predictions for the number of such halos and relics detectable with other low-frequency instruments are in the range of several hundred out to $z<0.6$ (Cassano et al. 2012).

At $150 \mathrm{MHz}$, the MWA will resolve 1-Mpc structures out to a redshift of 0.2 and $500 \mathrm{kpc}$ structures out to $z<0.07$. As with the detection of the cosmic web, confusion will be the major obstacle. Use of complementary higher resolution observations to model and subtract confusing sources will be required (Brown 2011). The TIFR GMRT Sky Survey (TGSS) that covers $32000 \mathrm{deg}^{2}$ north of declination $-30^{\circ}$ with angular resolution 20 arcsec presents a good comparison survey to the MWA for this work.

\subsection{Faraday tomography and magnetic fields}

The Milky Way and most other spiral galaxies all show wellorganised, large-scale magnetic fields, but the processes that amplify and maintain these fields are still not understood. On smaller scales, fluctuations in these magnetic fields regulate the dissipation of large-scale gas flows via a turbulent cascade. However, it is as yet unclear what processes generate turbulence in the interstellar medium (ISM), and how the subsequent spectrum and outer scale of turbulence depend on the surrounding conditions.

Polarised radio waves experience FR when they propagate through a magnetised plasma. The amount of rotation depends on the square of the wavelength, and on the path integral of $n_{\mathrm{e}} B_{\|}$along the line of sight (where $n_{\mathrm{e}}$ is the electron density of the ISM, and $B_{\|}$is the magnetic field strength parallel to the line of sight). By exploiting this phenomenon, the MWA will provide a new view of the large- and small-scale properties of otherwise unobservable magnetic fields in the Milky Way.

At the low observing frequencies of the MWA, small RMs have substantial effects on the distribution of polarised emission from the sky. This emission directly traces invisible foreground magnetic fields, which imprint their structure on the smooth polarised synchrotron emission from the Milky Way via foreground FR. By imaging the polarised sky at a range of frequencies, the MWA will provide direct measurements of the turbulent cascade in the local ISM, and will reveal the detailed magnetic geometries of discrete large-scale structures such as the Local Bubble, the North Polar Spur, and the Magellanic Clouds. Using the high spectral resolution of the MWA, we can also apply RM synthesis techniques (see also Section 2.4.1) to isolate overlapping RM components. An example is provided in Figure 5, which shows the turbulent pattern seen at $189 \mathrm{MHz}$ in a large region of the sky. The MWA will provide a unique tomographic view of magnetoionised gas, allowing the identification of multiple emitting and rotating regions along the same line of sight.

The MWA is expected to make the largest contributions to Galactic science by observing at mid- and high-latitude regions. Depolarisation is already severe in the inner Galactic plane at $1.4 \mathrm{GHz}$ (Reich, Reich, \& Fuerst 1990; Wolleben et al. 2006; Haverkorn et al. 2006) and it is likely that much of 


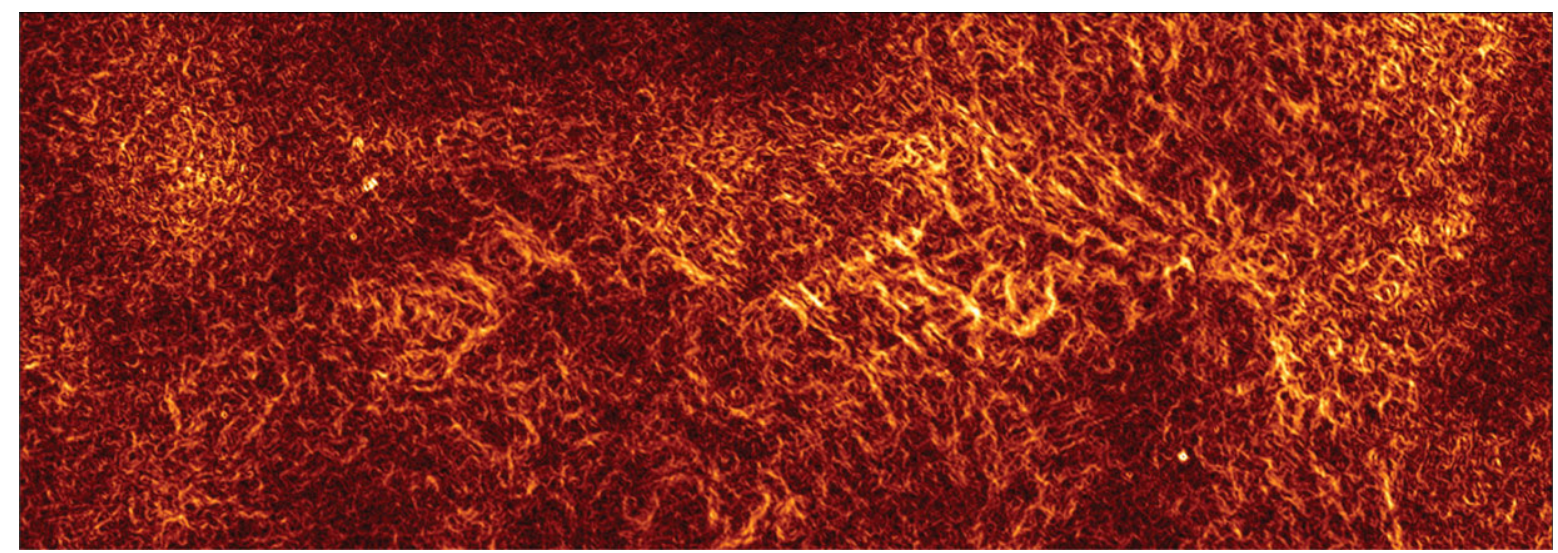

Figure 6. Gradient image of linear polarisation, $|\nabla \mathbf{P}|$, for an 18- $\mathrm{deg}^{2}$ region of the Southern Galactic Plane Survey (Gaensler et al. 2011) that has been used to study magnetised turbulence in the ISM. Similar statistical techniques will be applied to MWA polarised maps to investigate magnetic field strength, Mach number, Reynolds number, and other properties in the ISM.

the inner Galactic plane will appear completely depolarised below $300 \mathrm{MHz}$, although Bernardi et al. (2009) have detected polariased emission at arcminute scales at $150 \mathrm{MHz}$. Regions at mid and high latitudes, on the other hand, show considerable diffuse Galactic polarised structure at $350 \mathrm{MHz}$ (Haverkorn, Katgert, \& de Bruyn 2003b; de Bruyn et al. 2006; Schnitzeler, Katgert, \& de Bruyn 2009). The high sensitivity of the MWA to extended structure will enable it to map the diffuse polarised emission down to very low intensities, thus making visible weak Galactic magnetic fields in the local ISM and Galactic extended disc and halo that are undetectable at higher frequencies. RM synthesis across the entire MWA frequency band will result in an RM resolution of $0.3 \mathrm{rad} \mathrm{m}^{-2}$, which gives unprecedented detail in RM structure, allowing detailed studies of e.g. interstellar magnetised turbulence or magnetism in old supernova remnants. Increasing depolarisation as a function of decreasing frequency will give independent measurements of the amount and scale of fluctuations in the magneto-ionised medium (Sokoloff et al. 1998; Gaensler et al. 2001).

A powerful new additional probe of the magnetised ISM comes from the polarisation gradient, in which the spatial derivatives of Stokes $Q$ and $U$ are combined to reveal locations on the sky at which the electron density or magnetic field strength show sharp fluctuations (Gaensler et al. 2011). Application of the polarisation gradient at higher frequencies reveals a complex tangled network of narrow filaments (see Figure 6). These structures are a superset of the 'polarisation canals' seen in many radio polarisation images (Haverkorn \& Heitsch 2004; Fletcher \& Shukurov 2007; Schnitzeler et al. 2009), and directly reveal the locations of shocks, cusps, and shear in the turbulent ISM. The calculation of the polarisation gradient for magnetohydrodynamic simulations of interstellar turbulence shows that the intensity, alignment, and morphology of these filaments depend sensitively on input parameters such as the sonic Mach number and magnetic field strength (Burkhart, Lazarian, \& Gaensler 2012). By applying statistical metrics such as the genus and kurtosis, one can directly compare observations of the polarisations gradient with a grid of simulations, and thereby extract quantitative information on the otherwise unobservable turbulent parameters of the ISM.

Two effects distinguish the applicability of this technique for MWA data. First, the 'polarisation horizon' (Uyaniker et al. 2003) at the MWA's low observing frequencies is very nearby ( $\sim 100 \mathrm{pc}$, depending on observing frequency and Galactic coordinates; Bernardi et al. 2013), meaning that we can probe small-scale local features. Second, the change in electron density or magnetic field strength needed to produce a fluctuation in $Q$ or $U$ is very small (since the amount of FR is proportional to $\lambda^{2}$ ). For these two reasons, application of the polarisation gradient to MWA data should provide a detailed view of turbulence in the solar neighbourhood, and will be able to extend studies of the global turbulent cascade to smaller amplitudes and smaller angular scale than has been possible from previous observations.

RMs from extragalactic polarised point sources can be used to probe the intervening medium between the source and the observer, where generally the Galactic RM component dominates. Little is known about the polarisation percentages of extragalactic point sources at low frequencies. At $1.4 \mathrm{GHz}$, the expected number of linearly polarised sources down to a $3 \sigma$ sensitivity of $1 \mathrm{mJy}$ is about $1.2 \times 10^{4}$ per steradian (Beck \& Gaensler 2004). The number of polarised sources may decrease at low frequencies due to increased internal depolatisation. At 1.4 GHz, Mesa et al. (2002) found a median polarisation fraction of $2.2 \%$ for sources above $80 \mathrm{mJy}$ in the NVSS and Taylor, Stil, \& Sunstrum (2009) have compiled a catalogue from the NVSS of over 30000 polarised sources detected at better than $8 \sigma$ and selected for polarisation fraction larger than $0.5 \%$. The average polarisation fraction of sources in this catalogue is $\sim 7 \%$. At $350 \mathrm{MHz}$, Schnitzeler et al. (2009) found an average polarisation fraction of $3 \%$ for polarised sources brighter than $3 \mathrm{mJy}$ (see also Haverkorn, Katgert, \& de Bruyn 2003b). Bernardi et al. (2013) have surveyed $2400 \mathrm{deg}^{2}$ at $189 \mathrm{MHz}$ using 
the 32-element MWA development system. They achieved noise levels of $15 \mathrm{mJy}$ in polarisation and found only one polarised source, PMN J0351-2744, which had a polarisation fraction of $1.2 \%$ and an $\mathrm{RM}$ of $+34 \mathrm{rad} \mathrm{m}^{-2}$. The source was detected at $20 \sigma$. They also placed an upper limit of $\sim 1 \%$ on the polarisation fraction of over 100 other bright sources above $4 \mathrm{Jy}$ and detected diffuse polarised emission from the Galaxy with a peak level of $13 \mathrm{~K}$.

Beam depolarisation is likely to be partially responsible for the lack of detected sources by Bernardi et al. (2013) since the MWA demonstrator had only 15-arcmin resolution. Using $1 \%$ polarisation fraction as a lower limit, along with source flux counts from the 6C survey (Hales, Baldwin, \& Warner 1988) and the ratio of polarised sources to unpolarised sources of 14\% found by Banfield et al. (2011), we estimate the number of sources that will be detectable by the MWA assuming a fiducial sensitivity of $1 \mathrm{mJy}$ to be approximately $\sim 1$ source per square degree. These extragalactic source RMs will provide a 'grid' (Beck \& Gaensler 2004; Taylor et al. 2009) that can be used to model large-scale magnetic fields in the Milky Way and, if deeper observations can provide a sufficiently dense grid, in nearby galaxies. Provided that polarisation calibration is accurate enough, the MWA will observe point-source RM values with superb precision.

\subsection{The magellanic clouds}

The Magellanic System, consisting of the Large Magellanic Cloud (LMC), Small Magellanic Cloud (SMC), and other components such as the Magellanic Stream and the Bridge and Leading Arm, is the Milky Way's most significant neighbour. The Clouds and the Milky Way are engaged in a threebody interaction, allowing us to study at close hand the effect of dynamical interaction processes on the physical structure, star formation, magnetic fields, and chemistry of such galaxies. The location of the Clouds in the deep southern sky makes them ideal candidates to observe with the MWA in preparation for much more detailed studies at similar frequencies with the SKA.

A comparison of radio and far-infrared (FIR) emission in the Magellanic Clouds and other nearby galaxies allows for a more detailed study of the correlation of cosmic rays, magnetic fields, and star formation than is possible for distant galaxies. At GHz frequencies, ATCA and IRAS studies allowed Hughes et al. (2006) to find that the radio/FIR relation breaks down at $\sim 50 \mathrm{pc}(3 \mathrm{arcmin})$. Residual correlation at smaller scales remains due to the thermal component of the radio emission. Low-frequency observations of $6 \mathrm{C}$ galaxies have shown that the radio/FIR correlation remains good at $151 \mathrm{MHz}$ (Fitt et al. 1988). Such observations are useful for further separating thermal and non-thermal sources of emission, for the study of synchrotron spectral aging and cosmicray diffusion, and for allowing $k$-corrections in cosmological studies.
The Magellanic Clouds are among only a handful of galaxies where the magnetic field can be studied via both FR of the polarised emission from background sources (sensitive to the line-of-sight field) and the polarised component of the diffuse emission (sensitive to the field direction in the plane of the sky). Studies of the Clouds at $\mathrm{GHz}$ frequencies (Haynes et al. 1986; Gaensler et al. 2005a; Mao et al. 2008) have been useful in determining field strengths and morphology. Lowfrequency observations will contribute by locating regions, perhaps associated with wind-blown superbubbles, of warm ionised gas unseen in X-rays, and by allowing more detailed studies of Faraday structures. The MWA will investigate the existence of a possible pan-Magellanic magnetic field via the old relativistic electron population which will have travelled far from its source.

\subsection{Cosmic-ray mapping}

One of the most exciting possibilities of the MWA survey at the lowest frequencies is the mapping of the threedimensional cosmic-ray emissivity of the Milky Way. Within the MWA frequency range below approximately $200 \mathrm{MHz}$, Galactic H II regions turn optically thick, blocking emission from the synchrotron background of the Galaxy. Typical optical depths of $\mathrm{H}$ II regions are expected to be $\tau \approx 2-5$. These H II regions, therefore, appear in absorption relative to the surroundings and the residual surface brightness at these positions allows a direct measurement of the integrated emissivity of cosmic-ray electrons out to the $\mathrm{H}$ II region.

Nord et al. (2006) used 74-MHz VLA observations of H II regions to detect such absorption against the Galactic synchrotron background. They found 92 regions with absorption features, 42 of which have measured distances. While such features have been seen since the 1950s, previous observations had poor resolution. The 10 -arcmin VLA beam allowed for better spatial resolution while still remaining sensitive to low surface brightness. A number of surveys at higher frequencies have been conducted. Paladini et al. (2003) have compiled a catalogue of 1442 diffuse and compact H II regions based on 24 prior surveys, although a number of regions are omitted in their catalogue due to their emphasis on regions of the order of 10 arcmin (for an example of more recent identifications, see Bania et al. 2010). Approximately 800 of these regions have radio line velocity measurements providing distances. Paladini, Davies, \& De Zotti (2004) report on the distribution of the $\mathrm{H}$ II regions, most of which have Galactic latitude within $\pm 2^{\circ}$ of the plane and are distributed throughout the disc, with the spiral arm structure of the Galaxy evident in their distribution.

Because many Galactic $\mathrm{H}$ II regions have well-constrained distance estimates, MWA measurements of the synchrotron absorption features will allow for a three-dimensional map of the relativistic gas content in the Milky Way (e.g. Sun et al. 2008). For H II regions with kinematic distance degeneracies, MWA detections may be able to break the ambiguities. Since the MWA will have a view of the inner and outer Galaxy, it 


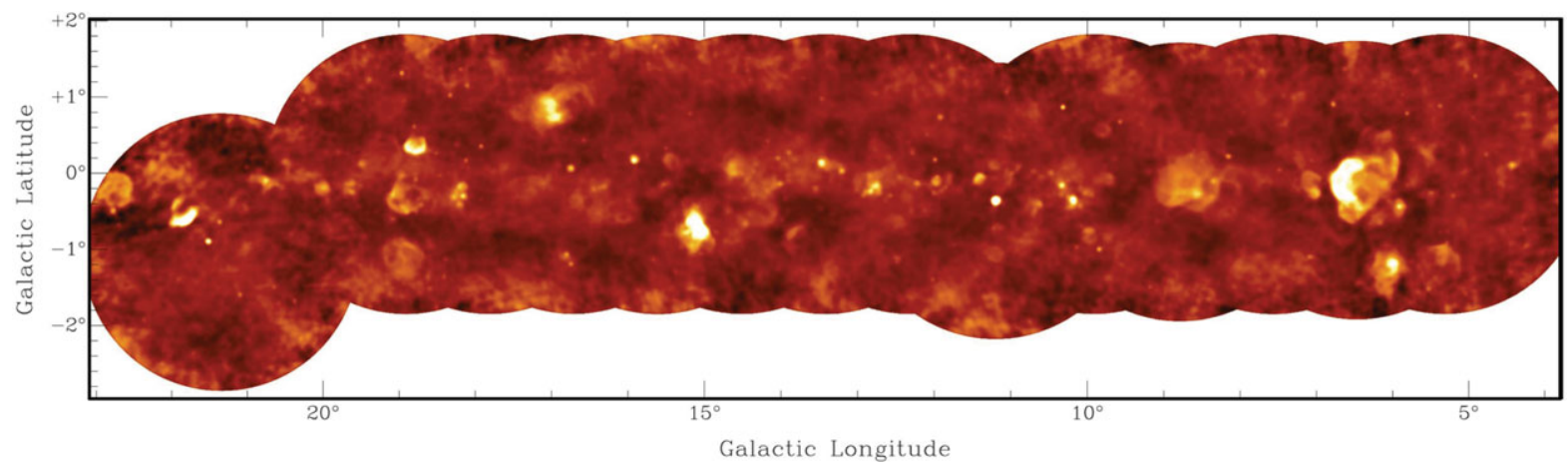

Figure 7. A 330-MHz panorama of part of the Galactic first quadrant, derived by smoothing VLA data from Brogan et al. (2006) to 3-arcmin resolution. At these low observing frequencies, most of the bright sources correspond to non-thermal emission from SNRs. The MWA will be able to carry out such observations over almost the entire inner Galaxy.

can provide the first accounting of the overall distribution of Galactic cosmic rays and of the large-scale magnetic fields that cause them to emit. In addition, due to its ability to measure the absorption in $\mathrm{H}$ II regions as a function of frequency, the MWA should be able to yield the cosmic-ray electron emissivity spectrum in the direction of the $\mathrm{H}$ II regions. As noted by Nord et al. (2006), multi-frequency observations should lead, therefore, to the mapping of the emissivity spectrum of cosmic rays.

Combined with the MWA's expected ability to detect and characterise supernova remnants (described below), these measurements will open new probes into the full energy budget of the Galaxy, including the role of supernovae in the production of energetic cosmic rays, as well as the diffusion and aging of the cosmic rays.

\subsection{Galactic supernova remnants}

Statistical studies of supernova rates suggest that there should be $\sim 1000-2000$ supernova remnants (SNRs) in our Galaxy (Li et al. 1991; Tammann, Loeffler, \& Schroeder 1994), in stark contrast to the $\sim 300$ SNRs currently known (Green 2004; Brogan et al. 2006). This deficit is likely due to the observational selection effects, which discriminate against the identification both of old, faint, large SNRs, and young, small SNRs. The discovery of these 'missing' SNRs, and hence a characterisation of the full SNR population, is crucial for understanding the production and energy density of Galactic cosmic rays and the overall energy budget of the ISM.

Recent studies have demonstrated that low-frequency interferometric maps are a very efficient way of identifying new SNRs, because of the comparatively steep radio spectra of these systems. Most notable has been the survey of Brogan et al. (2006), shown in Figure 7, which tripled the number of known SNRs in a 40-deg ${ }^{2}$ patch of the Galactic plane. The MWA will have a unique view of virtually all the inner Galaxy. The high-quality images that we expect to derive, across a range of frequencies, will provide a superb data set from which to identify hundreds of previously unidentified SNRs. Additionally, attempts to uncover old, faint sources will be enhanced by the development of new detection algorithms which we plan to implement on MWA data (e.g. circle Hough transforms; see Hollitt \& Johnston-Hollitt 2012). These sources will be of interest both as a statistical ensemble and because among them will be individual objects with unique or extreme properties.

\subsection{Radio recombination lines}

Low-frequency radio recombination lines (RRLs) are a sensitive tool for probing interstellar plasma. Below $100 \mathrm{MHz}$, carbon RRLs have been detected, but only in absorption, indicating an association with cold $(<50 \mathrm{~K})$ neutral gas or molecular clouds. At frequencies above $100 \mathrm{MHz}$, both carbon and hydrogen recombination lines have been observed, with the hydrogen lines likely associated with normal $\mathrm{H}$ II regions as at higher frequencies. Both carbon and hydrogen lines have been found in observations along the Galactic plane as far as $\pm 4^{\circ}$ off the plane in Galactic latitude.

Measurements of RRLs in the MWA frequency range will provide an opportunity to study both emission and absorption processes. They will enable new studies of diffuse photodissociation zones that lie between molecular clouds and the general ISM and yield estimates for electron temperature and density in these regions. Carbon RRLs at $75 \mathrm{MHz}$ exhibit a line-width range from 5 to $50 \mathrm{~km} \mathrm{~s}^{-1}$ (Erickson, McConnell, \& Anantharamaiah 1995), corresponding to $1-12 \mathrm{kHz}$. Although, the MWA spectral resolution is not ideal for such narrow lines, single-pixel RRL observations have previously been used with much success (Alves et al. 2012). Sensitivity can also be regained by stacking different transitions within the observing band.

\section{TIME-DOMAIN ASTROPHYSICS}

The systematic exploration of the time domain is becoming a dominant frontier in astronomy, and significant resources have been deployed across the electromagnetic spectrum in order to probe and understand transient and time-variable 
phenomena. Radio transients vary on time scales ranging from less than one second to many days. The sources are compact objects or energetic/explosive events, and each type is being investigated at the forefront of astrophysics.

Radio observations can provide keys to understanding the explosive and transient Universe by directly measuring the effects of magnetic fields and non-thermal processes that drive the outbursts (e.g. Cordes, Lazio, \& McLaughlin 2004). Even more intriguingly, synergies with multi-messenger astronomy (correlating electromagnetic transients with events detected in gravitational waves or neutrinos) have the potential to unlock substantial new physics. The MWA spans the upper frequencies associated with coherent radio sources and the lower frequencies of non-thermal magnetohydrodynamic processes. This makes observations of transients with the MWA particularly useful for understanding the nonequilibrium processes that drive dynamic astrophysical systems.

The potential sources of radio transient emission fall into five broad categories: coronal emission from nearby stars/substellar objects, emission from compact objects such as neutron stars and accreting black holes, explosive events such as gamma-ray bursts (GRBs) and radio supernovae, planetary emission from nearby systems, and new phenomena discovered through the opening of new regions of observational phase space. Of these, the first three are known sources of transient radio emission (at least at some frequencies), while the last two categories constitute more speculative targets. However, there are firm theoretical expectations that these latter objects should give rise to transient radio emission, and our searches will be far deeper than previous surveys.

First, we discuss in detail some of the expected physical constraints to be gained from the MWA observations of known sources of transients (Section 4.1). We then explore some of the more speculative science targets that we hope to explore with the new phase space opened up by the MWA (Section 4.2). We describe the new capabilities afforded by the MWA in Section 4.3 and in Section 4.4 we discuss observations of pulsars, which can be both steady and transient sources.

\subsection{Known transient targets}

\subsubsection{Low-mass stars and brown dwarfs}

Magnetised plasmas in stellar coronae (the outer layers of a star's magnetosphere) produce strong radio emission through non-thermal, collective, and/or coherent processes. This emission reflects local properties such as density and magnetic field strength and can be used to understand magnetic field generation, magnetospheric structure, angular momentum evolution, magnetic flaring mechanisms, and accretion processes. Furthermore, magnetic fields provide one of the few indirect probes of stellar interiors.
While radio studies of late-type stars and active binaries are well established (see Güdel 2002 for a review), radio observations of the lowest mass stars and brown dwarfsobjects incapable of sustained core hydrogen fusion (Kumar 1962) — have only recently been demonstrated and comprise a relatively new tool in this active field of research. $\mathrm{M}$ dwarfs with masses $0.1-0.5 \mathrm{M}_{\odot}$ are a dominant stellar component of the Galaxy, comprising $\sim 70 \%$ of all stars. They are also known to exhibit high levels of magnetic activity, with strong surface magnetic fields ( $\sim 2-4 \mathrm{kG}$, compared with $\sim 10 \mathrm{G}$ on the Sun; Saar \& Linsky 1985; Johns-Krull \& Valenti 1996) and substantial filling factors $(50 \%-80 \%$ of the surfaces of $\mathrm{M}$ dwarfs are covered by magnetic fields; Johns-Krull \& Valenti 1996). Magnetic radio emission from $M$ dwarfs has been observed in many nearby systems, both flaring and quiescent, predominantly in the $1-10-\mathrm{GHz}$ band. These studies have confirmed the existence of large-scale, organised magnetic fields around stars with fully convective interiors (Donati et al. 2006), despite the loss of the $\alpha \Omega$ dynamo that powers the Solar field (Parker 1955).

For even lower mass stars and brown dwarfs, the detection of both quiescent and flaring radio emission was initially a surprise (Berger et al. 2001), as the flux from these sources is orders of magnitude greater than that expected from the Güdel-Benz relation. ${ }^{5}$ Several late-type sources have now been detected at $\mathrm{GHz}$ frequencies (Figure 8), in many cases despite the absence of optical or X-ray emission (see Berger 2006 for a review). Indeed, while $\mathrm{H} \alpha$ and X-ray luminosities relative to bolometric luminosities appear to decline with spectral type, relative radio emission inexorably increases up to the substellar regime (see Burgasser \& Putman 2005; Berger 2006; Audard et al. 2007). Understanding why radio emission is so prominent among these very low mass objects, and whether such emission correlates with age, rotation, or other physical parameters, remain outstanding questions.

Radio flares in particular demonstrate that this emission is associated with magnetic events, occurring over timescales of microseconds to minutes (e.g. Osten \& Bastian 2006). The brightness temperatures of bursting emissions frequently exceed $10^{9} \mathrm{~K}$, indicating coherent processes as confirmed by high circular polarisations $(30 \%-100 \%)$. Yet, radio variability from these sources is remarkably diverse, from strong (30-mJy peak flux), narrowband, fully polarised, bursting emission with frequency drifts (Burgasser \& Putman 2005), to periodic bursts suggestive of pulsar-like beaming (Hallinan et al. 2007, and Figure 8), to order-of-magnitude variations spanning years (Antonova et al. 2007). It has even been suggested that the observed 'quiescent' emission from these sources may be the result of low-level, sustained electron cyclotron maser emission (e.g. Hallinan et al. 2007). The physical origin of transient radio bursts from low-mass stars, their incidence rates (duty cycle estimates range from

\footnotetext{
${ }^{5}$ A correlation between radio flux and X-ray luminosity that extends over several orders of magnitude; see Güdel \& Benz (1993).
} 


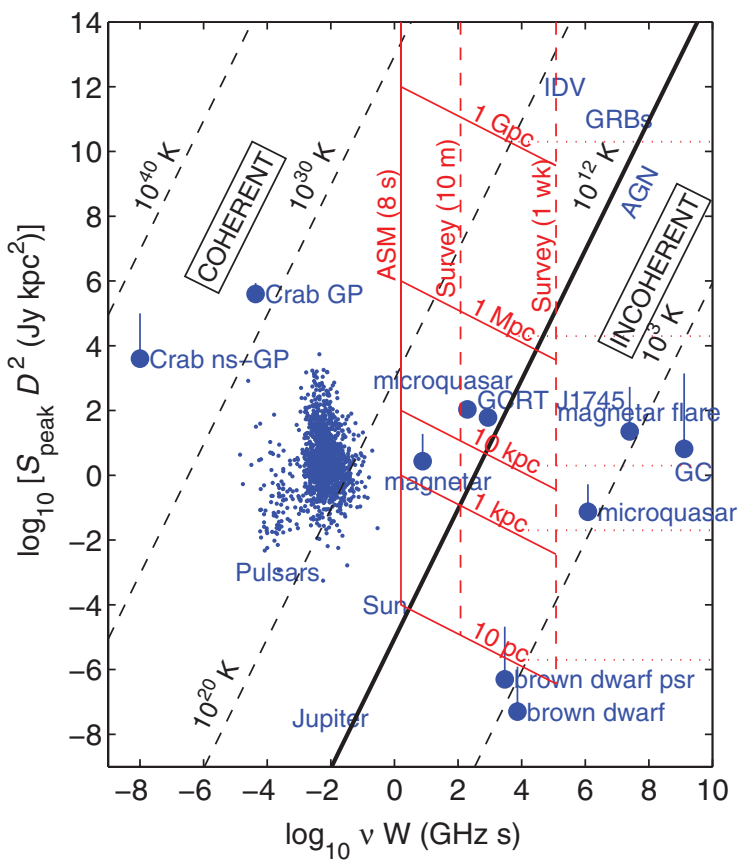

Figure 8. Phase space for radio transients observable with the MWA at a nominal frequency of $200 \mathrm{MHz}$, updated and adapted from Cordes et al. (2004). We plot the product of the observed peak flux density $S_{\text {peak }}$ and the square of the distance $D^{2}$ (like a luminosity) against the product of the emission frequency $v$ and the transient duration $W$. In the Rayleigh-Jeans approximation, these quantities are directly proportional and related to the brightness temperature $T$ (given by the diagonal lines); we use a brightness temperature of $10^{12} \mathrm{~K}$ (thick diagonal line) to divide coherent and incoherent processes (Readhead 1994). The red lines show the predicted sensitivity of the MWA, assuming a 50-mJy source can be detected in $8 \mathrm{~s}$. The vertical red lines give the timescales for individual snapshot observations ( $8 \mathrm{~s})$, and shortand long-term surveys ( $5 \mathrm{~m}$ and 1 week). The red diagonal lines give the $S_{\text {peak }} D^{2}$ limits corresponding to distances of $10 \mathrm{pc}$ (appropriate for sources such as low-mass stars, brown dwarfs, and planets), $1 \mathrm{kpc}$ (local Galactic sources), $10 \mathrm{kpc}$ (the Galactic Center), $1 \mathrm{Mpc}$ (local group), and $1 \mathrm{Gpc}$ (extragalactic sources); the dotted lines show an estimated confusion limit of $10 \mathrm{mJy}$, although we can search below this limit using image subtraction or for polarised emission.

$1 \%-30 \%$ ), and their spectral characteristics are either poorly constrained or entirely unknown. These questions have a direct impact on whether low-mass stars comprise a significant fraction of radio transients in general (e.g. Kulkarni \& Phinney 2005; Kaplan et al. 2008).

The MWA has the potential to contribute substantially to our understanding of both quiescent and flaring magnetic emission from low-mass stars and brown dwarfs. By sampling the $80-300-\mathrm{MHz}$ band, MWA observations can be used to constrain the peak frequency $\left(v_{\mathrm{pk}}\right)$ and spectral indices about $v_{\mathrm{pk}}$, enabling the determination of coronal field strengths (currently estimated at 10-100 G; Burgasser \& Putman 2005; Berger 2006), electron densities and the fundamental nature of the emission. Audard et al. (2007) argue that radio emission from very low mass stars may in fact peak in the $\sim 100-\mathrm{MHz}$ range if it arises from electron cyclotron emission from weak fields $\left(v_{\mathrm{pk}}=v_{c}=30-300 \mathrm{MHz}\right.$ for 10
100-G fields; see also Osten et al. 2006). The sensitivity of the MWA is essential for this work, given the intrinsic faintness, but variable nature, of radio emission from low-mass stars; estimates range over $10 \mu \mathrm{Jy}$ to $100 \mathrm{mJy}$ at $100 \mathrm{MHz}$ at a distance of $10 \mathrm{pc}$, depending on the emission mechanism. Similarly, the MWA's wide-field capabilities are necessary to build up statistically robust samples, particularly given the roughly uniform distribution of nearby low-mass stars in the vicinity of the Sun. High spectral resolution observations enabled by the MWA will permit studies of field dynamics and remote determinations of physical parameters such as the heights of magnetic reconnection regions, electron speeds, and the density profile of the stellar coronae.

\subsubsection{Magnetars}

While a variety of neutron stars can produce transient or steady radio emission (discussed in more detail in Section 4.4), some subclasses of neutron stars can give rise to extremely energetic, explosive phenomena. With magnetic fields of $\sim 10^{15} \mathrm{G}$, magnetars (Duncan \& Thompson 1992; Thompson \& Duncan 1995) undergo catastrophic reconnection events known as 'giant flares' that release $>10^{43} \mathrm{erg}$. With each flare the magnetars release blobs of relativistic plasma (Frail, Kulkarni, \& Bloom 1999; Gaensler et al. 2005b; Cameron et al. 2005) with peak fluxes of hundreds of mJy at frequencies from $200 \mathrm{MHz}$ up to $100 \mathrm{GHz}$ (Figure 8). Such events are detected through high-energy satellites, but the radio monitoring provides vital diagnostics of the flare properties and energetics.

Beyond this, some magnetars unexpectedly have steady radio emission that is modulated at the $\mathrm{X}$-ray pulse period (2-12 s). Prior searches for steady-state radio emission from magnetars had not been successful (e.g. Kaplan et al. 2002), but Halpern et al. (2005) found a radio source coincident with the magnetar XTE J1810-197 (also Camilo et al. 2006, and others). Monitoring the magnetars through both individual pulses and phase-averaged emission, looking for both giant flares and the onset of bright phases, will help understand the duty cycles of the radio-bright phases and hence unravel the puzzles that they present.

\subsubsection{X-ray binaries}

Relativistic outflows or jets are a widespread yet transient consequence of accretion onto compact objects. Radio jets are seen for black-hole binaries (BHBs) and some neutronstar binaries (NSBs). These sources are collectively called 'microquasars' (Figure 8), since they exhibit scaled-down and rapidly evolving versions of the jets in active galactic nuclei. Microquasars exhibit several types of jets with different ranges of radio power and ejection timescale. Their study is crucially dependent on the correlated variations in radio and $\mathrm{X}$-ray properties.

The most common type of jet in microquasars is the quasisteady jets associated with the X-ray hard state (Fender, Belloni, \& Gallo 2004; McClintock \& Remillard 2006) that can persist for months in some sources. Steady jets are 
estimated to be mildly relativistic (i.e. $\sim 0.7 c$; see Fender 2006), but there is uncertainty about the baryonic content and hence the scale of the mass outflow and the fraction of accretion energy needed to power such jets.

Impulsive ejections are also produced by BHBs and some NSBs and the brightest of these are seen as bipolar radio jets that move with velocities as high as $0.98 c$ (Mirabel \& Rodríguez 1999). Impulsive jets are associated with X-ray state transitions (Fender et al. 2004). In two BHBs, superluminal radio ejections, coinciding with major X-ray flares, led to the appearance of X-ray jets (more than one year later), that were imaged with Chandra as the jets impacted the local ISM (Tomsick et al. 2003; Corbel et al. 2005). Smaller and more frequent ejections in BHBs are seen as correlated X-ray and radio flares with $\sim 10$-min timescales and radio-band delays (Eikenberry et al. 2000; Wilms et al. 2007). The available X-ray data usually reveal recurrent and abrupt changes in flux and spectral components that associate the small jets with instability cycles in the inner accretion disc.

Study of the connection between discs and jets is fundamentally based on correlated X-ray and radio behaviour, as outlined above. During the last decade, the primary limitation for this enterprise has been the absence of dedicated radio instruments that can partner with $\mathrm{X}$-ray facilities, but the MWA will radically change this landscape. Additional partners for jet investigations arise with Fermi (10 keV to $300 \mathrm{GeV}$ ) and the recent expansions of $\mathrm{TeV}$ observatories (e.g. HESS and VERITAS; $50 \mathrm{GeV}$ to $50 \mathrm{TeV}$ ).

Recent measurements with the GMRT (150-1450 MHz) in India have shown variable radio emission in GRS $1915+105$ (see Figure 8; Ishwara-Chandra et al. 2005) and Sco X-1 (Pandey et al. 2005), with spectra that do not exhibit selfabsorption cutoff at low frequencies. This is interpreted as emission from the outer regions of a compact jet and from an optically thin impulsive jet, respectively (also see Pandey et al. 2006, 2007).

Overall, the MWA has the capability to monitor many known sources for which X-ray activity is detected, establishing outburst statistics and extending the spectra down to low frequencies where the total energetics are constrained. These observations can help to measure the burst luminosity function and the X-ray/radio correlation, and investigate their dependences on various intrinsic source parameters such as mass and X-ray luminosity. The MWA will provide data archives for the two microquasars that are reliable sources of jets: SS 433 and GRS 1915+105, as well as the BHBs and NSBs with the fastest outburst recurrence time (1-2 years: 4U 1630-47, and GX339-4, Aql X-1, 4U 1608-52, and the rapid burster). These monitoring observations will also find new outbursts of known sources, acting as a trigger for observations across the electromagnetic spectrum. Finally, the MWA has the potential to discover new sources: the large majority of the known BHBs and candidates are transient sources that were discovered because of their X-ray outbursts (Remillard \& McClintock 2006).

\subsection{Serendipitous transient sources}

Beyond the source categories discussed above, there are a number of object classes which we might hope to see (see Cordes et al. 2004; Fender et al. 2006). These vary from the local to the cosmological, and the population predictions also span a wide range. Currently, low-frequency transient radio emission has been proposed but not observed for these objects, and there are only limited observational constraints. Such sources are among the most exciting possibilities for the MWA transient surveys (Section 4.3). Those surveys cover a large area of unexplored phase space-orders of magnitude in several different parameters - and provide a real opportunity to discover unique sources.

\subsubsection{Extra-solar planets}

In the Solar System, Jupiter is a strong source of lowfrequency radio emission produced by cyclotron maser processes in its magnetosphere (Gurnett 1974; Wu \& Lee 1979). Observations have established that many of the Jovian planets discovered around other stars (e.g. Marcy et al. 2005) have magnetic fields (Shkolnik, Walker, \& Bohlender 2003), although the field strengths are not known. By analogy, many of those planets should have similar emission. This would then be a novel way of directly detecting the planetary companions, something that has only been possible in a few special cases otherwise (Charbonneau et al. 2002). This would allow an unprecedented probe of the planetary systems, such as estimating properties of the planetary magnetosphere, measuring its rotation rate, and observing the interaction between the star and planet (Lazio et al. 2004). Grießmeier, Zarka, \& Spreeuw (2007) discuss theoretical expectations for radio emission from known extrasolar planets. Most of the sources are predicted to emit at the microJansky level between 10 and $200 \mathrm{MHz}$. A handful of planets are expected to emit at the mJy level in the MWA bandpass. Their results are heavily model dependent and MWA observations of the currently known exoplanet population will help constrain further models.

There have been a number of searches for emission from such planets (e.g. Farrell, Desch, \& Zarka 1999; Bastian, Dulk, \& Leblanc 2000; Zarka et al. 2001; Lazio et al. 2004; Lazio \& Farrell 2007). There are no detections to date, although the results are not very constraining. The emission is predicted to be very sporadic and bursty, and it is difficult to search effectively for such emission with pointed instruments like the VLA. Instead, new instruments like the MWA are likely to be the best way to detect extra-solar planets (Lazio et al. 2004).

\subsubsection{Explosive events}

GRBs and radio supernovae may both produce short- and long-duration transient radio signals. The afterglow emission from each type will provide signals at the upper frequencies accessible to the MWA that are delayed from the initial explosion (due both to plasma in the ISM and to changing 
opacities) and slowly brighten over a few weeks to months (Morales et al. 2005). These afterglows can be important diagnostics for explosion energetics (Soderberg et al. 2007, 2010) and can help understand particle acceleration in the nearby Universe (Chakraborti et al. 2011). While the long timescales of such events at low frequencies make the MWA unfavourable for their discovery, it will be able to study afterglows of events identified initially at higher frequencies.

GRBs, supernovae, and other explosions may also produce prompt pulses of coherent emission during the initial explosion (Colgate \& Noerdlinger 1971; Nakar \& Piran 2011). The theorised prompt signals are produced by coherent emission near the explosive shock. Proposed mechanisms include current oscillations (Usov \& Katz 2000), synchrotron maser activity behind the shock (Sagiv \& Waxman 2002), or collective plasma modes as seen in solar bursts (Moortgat \& Kuijpers 2005). Models by Hansen \& Lyutikov (2001) and Benz \& Paesold (1998) also predict detectable emission, depending on the plasma environment and dynamics of the GRB. Some of these signals will be accompanied by bursts of radiation at other wavelengths, while some may offer the potential for multi-messenger astronomy by correlating radio and gravitational-wave signals (Nakar \& Piran 2011; Palenzuela, Lehner, \& Liebling 2010).

The detection of such prompt emission from GRBs offers a number of unique insights into the properties of the bursts, the intergalactic medium, and cosmology (Morales et al. 2005). First, the details of the emission that we see depend heavily on the specific model for the shock structure, as discussed above. Second, we can use the emission as a probe of the media through which it propagates: we will see delays and dispersion from the local plasma, the host galaxy's ISM, the intergalactic medium (details of which can depend on cosmic reionisation), and the Milky Way's ISM (Inoue 2004).

Past searches for prompt radio emission from GRBs (Dessenne et al. 1996; Benz \& Paesold 1998; Balsano 1999) were all unsuccessful. Other projects attempted to perform blind surveys for radio transients (Amy, Large, \& Vaughan 1989; Baird et al. 1976; Katz et al. 2003). All of these attempts have suffered from constraints inherent in narrowband receivers and simple transient identification systems. More modern projects like GASE and ETA (Morales et al. 2005; Patterson et al. 2009) were designed to correct many of these problems, with digital baseband recording and interference rejection through de-dispersion, but the collecting areas are still very small.

\subsubsection{New opportunities}

Above we discussed two classes of sources that are expected to emit low-frequency radio transients, and where the detection of such transients would enable us to make significant advances in our understanding of such objects, but where we do not know the source properties enough to consider them 'guaranteed' science. Beyond even those classes are additional objects that may be found with blind searches (e.g. neutron star binary inspiral events that may produce radio precursors; Hansen \& Lyutikov 2001).

To date, systematic transient searches with radio telescopes have yielded some results, but they have largely been difficult to interpret (e.g. Matsumura et al. 2007, 2009), due to poor statistics, limited localisation, and a long time before followup. However, there have been successes that, while enigmatic, hint at the potential offered by the MWA. We do not give a full review here, but point to particular detections that hold promise for the MWA.

While searching for transient events at the Galactic Centre (GC), Hyman et al. (2002) found two sources, one of which is an X-ray binary, but the other remains unclassified, although it varies on long timescales ( $\sim$ months). Later searches (Hyman et al. 2005, also see Hyman et al. 2009) found a much more rapidly variable source, with flares on timescales of minutes that repeated (sometimes) every $77 \mathrm{~min}$ (GCRT J1745 in Figure 8). The positional coincidence with the GC, along with the greatly increasing source density near the Centre, suggests that it is at a distance of about $8 \mathrm{kpc}$, but this could be a selection effect because all the fields searched for transients in the programme were in that direction. Bower et al. (2005) found another transient near the GC, but they deduced that it was an X-ray binary with a jet outburst interacting with the dense medium in that region.

For searches of high-latitude fields, Bower et al. (2007) followed by Frail et al. (2012) searched archival VLA data at $\mathrm{cm}$ wavelengths and identified at least one very faint (sub-mJy) transient source. Jaeger et al. (2012) did repeated observations at a frequency of $325 \mathrm{MHz}$ (closer to the MWA band) and did identify one significant transient of unknown origin, although they consider several possibilities including a distant flare star. Only recently have surveys moved beyond examinations of archival data (e.g. Bannister et al. 2011), with a wide range of flux limits and timescales, to systematic examinations of the variable sky (e.g. Lenc et al. 2008; Croft et al. 2011). It is becoming clear that radio transients are rare but promising targets that will lead to advances in a number of areas of astrophysics.

\subsection{Planned searches}

We will conduct a continuous search for transient radio emission in each image produced by the MWA's real-time system, looking for changes from the previously observed sky on timescales ranging from $8 \mathrm{~s}$ to months (eventually using the high-quality integrated data from the sky surveys). This search will be more than six orders of magnitude more sensitive than previous transient surveys in the band and will cover a much broader range of frequencies, transient durations, and dispersion delays.

Eventually the full transient capabilities of the MWA will (1) cover 0.25 steradian instantaneously-approximately $\sim 3 \%$ of the visible sky-with full polarisation coverage; (2) search blindly over that area orders of magnitude deeper than previous work; (3) simultaneously record light curves 


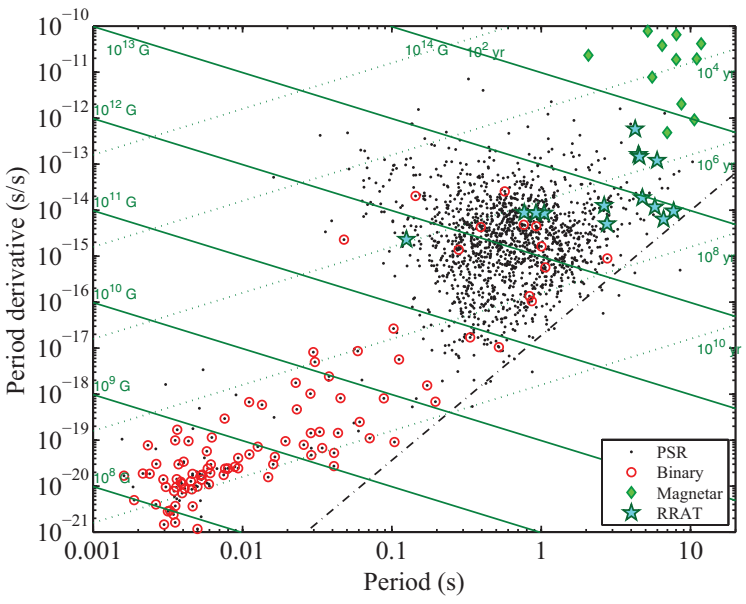

Figure 9. $P-\dot{P}$ diagram showing the distribution of the known pulsar population. The diamonds show the magnetars (SGRs and AXPs) and the stars the RRATs; binary systems are circled. The diagonal lines show loci of constant dipole magnetic field (solid) and spin-down age (dotted), while the dot-dashed line is an approximate 'death-line' for pulsar activity. The MWA will be uniquely sensitive to the slower, transient population while maintaining sensitivity to a considerable fraction of the millisecond pulsar population in the lower left.

and spectra for hundreds of sources and conduct routine observation of tens of thousands of sources per week; (4) obtain typical exposures of days/source, with hundreds to thousands of hours for sources in selected areas (Galactic plane and EOR fields) over one year; and (5) record full electric field time series for a subset of observations.

New sources detected by the MWA will have relatively poor (approximately arcminute) localisation. This will hinder interpretation as many possible counterparts will be present at other wavelengths (e.g. Kaplan et al. 2008). Prompt multiwavelength follow-up with wide-field imaging instruments will be crucial to help mitigate this, as the detection of contemporaneous variability at other wavelengths will significantly improve the localisation of sources and will pave the way for more detailed follow-up observations such as spectroscopy.

Source confusion will limit the sensitivity of the MWA for source detection. However, time variability is a classic way to help beat the confusion limit (i.e., searches for pulsars with single-dish telescopes). Subtracting images of the sky taken over successive time periods will help beat down the confusion noise and allow transients to emerge, especially when correlated with observations at other wavelengths. Finally, the examination of polarised images that have much lower background source levels will allow searches for bright and highly polarised transients like GCRT J1745-3009 (Hyman et al. 2005).

\subsection{Pulsars}

With approximately 2000 now known (Figure 9), pulsars offer opportunities to study a range of astrophysical phe- nomena, from the microscopic to the Galactic. The MWA's wide coverage in frequency and large collecting area makes it a unique instrument for low-frequency detection and detailed studies of pulsars and fast transients. The areas of pulsar science that the MWA would probe at low frequencies include pulsar phenomenology and pulsar searches, in general, and single-pulse and giant pulse studies, in particular. This is highlighted by the successful detection of giant pulses from the Crab Nebula pulsar at $200 \mathrm{MHz}$ with only three prototype MWA antenna tiles (Bhat et al. 2007).

The MWA will be able to observe at rates faster than the $0.5-8 \mathrm{~s}$ correlator integrations using a voltage capture system capable of recording, for several hours, correlator input data. This system will provide Nyquist sampled voltage streams for $3072 \times 10 \mathrm{kHz}$ channels comprising the full instantaneous bandwidth from each of the 128 antennas of the MWA that will enable high time-resolution studies of several types of objects, including pulsars and fast transients over the $\sim 1000 \mathrm{deg}^{2}$ tile beam.

It is at MWA frequencies that pulsar emission peaks (Maron et al. 2000; Kondratiev et al. 2012) and that interstellar propagation effects are easiest to study, but this wavelength range has been neglected for years because of difficulties in processing the data and the increasing prevalence of radio frequency interference (RFI). This has left the lowfrequency pulsar population largely unconstrained. Since low frequencies select against distant objects (especially for fast pulsars), the MWA will provide important new constraints for the local, low-frequency population. As a baseline, in an 8-h pointing at $200 \mathrm{MHz}$ we should have a $8 \sigma$ sensitivity limit of $1.8 \mathrm{mJy}$ for long-period pulsars. When corrected to 1400 $\mathrm{MHz}$ using a spectral index of -1.8 (Maron et al. 2000), this would be $0.05 \mathrm{mJy}$, comparable to or better than ongoing surveys like the High Time Resolution Universe survey (Keith et al. 2010) or the Green Bank 350-MHz Driftscan Survey (Boyles et al. 2013; Lynch et al. 2013).

Studies of individual pulsars help address a number of areas of astrophysics, providing unique insight into the asyet poorly understood pulsar emission mechanism and astrophysical plasmas (Melrose 2003). Assembling an average pulse profile from thousands of individual pulses can help investigate the emission further, probing the emission altitude, beam geometry, and inclination of the magnetic field from the spin axis (Jenet, Anderson, \& Prince 2001; Gupta \& Gangadhara 2003; Kondratiev et al. 2012; Hassall et al. 2012). Statistics derived from average pulse profiles may be among the best probes of complicated phenomena like core-collapse supernova (e.g. Johnston et al. 2005). Longer timescale variations in the emission can directly constrain the density of the magnetospheric plasma (Kramer et al. 2006).

Single-pulse studies should be possible ${ }^{6}$ for a sample of pulsars in excess of 100, with the full MWA system at

\footnotetext{
${ }^{6}$ The fractional magnitude of pulse-to-pulse variation in the conal profiles is expected to be close to unity. Therefore, although desirable, it is not crucial to have the ability to detect individual pulses from the sequences for assessing primary parameters of these fluctuations, since the detection
} 
frequencies $>100 \mathrm{MHz}$ (Kondratiev \& the LOFAR Pulsar Working Group 2012). In cases of bright pulsars, we aim to probe the polar emission regions at different heights in greater details to study single-pulse fluctuations simultaneously across a wide range of radio frequencies, believed to come from different heights from the star surface. Mapping of the emission patterns corresponding to different heights simultaneously will enable tomography of the pulsar emission cone (Maan et al. 2013; Asgekar \& Deshpande 2001; Deshpande \& Rankin 1999). The wide bandwidth of MWA can probe a large fraction of the emission cone, and at larger distances from the star than higher frequency telescopes, revealing much needed clues about the mechanism of pulsar radio emission, which is still poorly understood even after 40 years of pulsar studies.

\subsubsection{Rotating radio transients}

While observations of pulsars with regular steady pulses reveal much about those sources, it is in sources where the pulsar emission becomes irregular that we can hope to gain disproportionate insight about the pulsar emission mechanism and the relation of pulsars to other more exotic classes of neutron stars. In particular, the rotating radio transients (RRATs; McLaughlin et al. 2006; Figure 9) likely serve as links to other more extreme populations. The RRATS emit only sporadic dispersed radio bursts of between 2 and $30 \mathrm{~ms}$ in duration that are separated by $4 \mathrm{~min}$ to $3 \mathrm{~h}$, but nonetheless are identifiable as rotating neutron stars by their long-term spin-down. Initial estimates suggest that there could be as many RRATs as traditional pulsars (McLaughlin et al. 2006; Keane et al. 2010), but their properties do not appear the same, as they occupy different parts of the $P-\dot{\mathrm{P}}$ diagram (see Figure 9).

The study of RRATs and sources with and without radio emission may help link the canonical rotation-powered pulsars-neutron stars that emit primarily in the radio and that are powered by rotational energy - to other classes of neutron stars that are powered via other energy sources. In particular, the $\mathrm{X}$-ray and $\gamma$-ray sources known as magnetars (Woods \& Thompson 2006), which manifest observationally as the currently ${ }^{7} 11$ confirmed anomalous X-ray pulsars (AXPs) and 9 soft gamma-ray repeaters (SGRs), are powered by the decay of superstrong $\left(\gtrsim 10^{14} \mathrm{G}\right)$ magnetic fields. In contrast, the soft X-ray sources known as isolated neutron stars (INS; Kaplan 2008) are powered by residual heat, but may be the decayed remnants of old magnetars (Kaplan \& van Kerkwijk 2009). There is currently a great deal of interest in linking radio pulsars to these populations. At least two magnetars have been seen as transient radio pulsars (Camilo et al. 2006, 2007), while radio searches for the INS have so far been negative (Johnston 2003; Kaplan, Kulkarni, \& van

of relatively stable fluctuation features/properties benefits from the total integration time in quite the same way as the average profile estimation does.

${ }^{7}$ http://www.physics.mcgill.ca/ pulsar/magnetar/main.html
Kerkwijk 2003; Kondratiev et al. 2009). The populations may be linked via RRATs: the X-ray and timing characteristics of the RRATs offer tantalising clues to their relation to both magnetars (Lyne et al. 2009) and the INS (Popov, Turolla, \& Possenti 2006; McLaughlin et al. 2007), but as yet nothing firm has been established (Keane \& Kramer 2008).

The identification of RRATs relies on detecting single, dispersed pulses rather than steady repeating pulses (as in Keane et al. 2011; Burke-Spolaor \& Bailes 2010). They do appear as repeating transients and so many of the techniques required will be similar to those for other transients (Lorimer et al. 2007; Keane et al. 2012). We will also explore the bispectrum techniques of Law \& Bower (2012), which enable computationally simple searches for dispersed pulses at the expense of $\mathrm{S} / \mathrm{N}$, although the small area of each antenna leads to a low $\mathrm{S} / \mathrm{N}$ on each baseline, making such searches (Law et al. 2012) significantly less sensitive than traditional beamforming.

\subsubsection{Interstellar medium}

Beyond the study of pulsars themselves, variations in individual pulses with time and frequency can be used to probe the properties of the ISM (Hassall et al. 2012) and the Galactic magnetic field (Noutsos et al. 2008), as well as the solar corona (Ord, Johnston, \& Sarkissian 2007). Signals of short intrinsic duration do not survive passage through interplanetary, interstellar, or intergalactic plasma unscathed (Rickett 1990; Cordes et al. 1991; Taylor \& Cordes 1993) and are distorted via dispersion (Cordes \& Lazio 2002), scintillation (Rickett 1969), scattering (Bhat et al. 2004), and FR.

Dispersion makes lower frequencies arrive later. Compared with a signal at infinite frequency, a signal of frequency $v$ arrives with a time delay $\propto v^{-2}$. Dispersive delay can be both an advantage and a disadvantage. A radio counterpart to a transient event, such as a GRB, observed with a high-energy satellite, would be delayed by several minutes to hours before it arrives in the MWA band. This delay is beneficial since it allows time to prepare for the event at low frequencies (e.g. Cordes \& McLaughlin 2003; Macquart 2007). But the converse is also true. If the MWA provides the first detection of a transient signal, it may be too late for other facilities at higher frequencies to observe it.

Scattering is the result of multi-path propagation in ionised media. While it has other effects, such as spectral and angular broadening, we are primarily concerned with temporal broadening here. This leads to information on timescales shorter than the scattering time being lost, and this time scales as $\propto v^{-22 / 5}$. Because of this steep scaling, this tends to set a minimum useful timescale for low-frequency observations. The scattering timescale becomes comparable to the standard MWA correlator integration time of $0.5 \mathrm{~s}$ in the Galactic plane for objects beyond $\sim 5 \mathrm{kpc}$ (Cordes \& Lazio 2002). Hence, the MWA will be most sensitive to scattering effects for sources within this distance. 


\section{SOLAR, HELIOSPHERIC, AND IONOSPHERIC SCIENCE}

The MWA will make significant contributions to the fields of solar, heliospheric, and ionospheric science through a diverse set of novel radio measurements, as described here and in earlier papers (Bastian 2004; Cairns 2004; Oberoi \& Kasper 2004; Salah et al. 2005; Oberoi \& Benkevitch 2010). These measurements directly address space weather, defined as the study of the impact of the Sun on the Earth and its neighbourhood. Space weather effects are mostly driven by solar flares and coronal mass ejections (CMEs) that produce electromagnetic radiation (from X-rays to radio wavelengths), energetic particles, shock waves, changes in magnetic fields, and fast-moving plasma outflows that couple to the magnetosphere, ionosphere, neutral atmosphere, and surface of the Earth. These responses can directly affect humanity's environment, technology, and society (Scherer et al. 2005; Warmuth \& Mann 2004). The MWA will be able to observe and track CMEs, shocks, and other plasma structures from points close to their inception through their journey to the Earth and beyond, and it will be able to characterise their impact on the terrestrial ionosphere, making it a powerful instrument for space weather studies.

\subsection{Solar science}

Even the quiet radio Sun is a time-variable, spectrally and morphologically complex, extended source. Emission features are especially remarkable during periods of high solar activity. The active Sun produces both broadband $(\Delta f / f \approx$ 1) and narrowband $\left(\Delta f / f \ll 10^{-2}\right)$ emissions at frequencies from below $1 \mathrm{MHz}$ to approximately $30 \mathrm{GHz}$ (e.g. Wild, Smerd, \& Weiss 1963; Bastian, Benz, \& Gary 1998; Cane, Erickson, \& Prestage 2002; Karlický 2003; Cairns 2011). Variations in the emission can occur over timescales ranging from less than $1 \mathrm{~ms}$ to the 11-year cycle of solar activity. The complexity of solar radio emission presents a challenging radio imaging problem, but the unprecedented instantaneous dynamic range, wide bandwidth, high spectral resolution, and full-Stokes capability of the MWA will enable spectroscopic imaging of the dynamic Sun.

The physical sources of solar radio emission in the MWA band fall into three distinct categories: (1) thermal emission produced by hot electrons in all layers of the solar atmosphere, but especially the solar corona (McLean \& Labrum 1985); (2) gyro-synchrotron and synchrotron emission with broad power-law spectra in frequency, created by electrons accelerated in magnetic loops and by shocks driven by CMEs (Bastian et al. 2001); and (3) intense bursts of nearly coherent narrowband emissions that are produced as the so-called type I, II, III, IV, and V bursts (e.g. Wild et al. 1963; McLean \& Labrum 1985; Cane et al. 2002; Cairns 2011). The presence of multiple different emission mechanisms, along with propagation effects like refraction and scattering, endows ra- dio observations with a rich and unique coronal diagnostics capability.

\subsubsection{Electron density and temperature}

Large gradients in electron density and temperature from the solar chromosphere to the coronal regions have the consequence that the optical depth to radio waves tends to be a sensitive function of the ray path, which itself is determined by the refraction due to the density gradients. The optical depth of ray paths at different frequencies is dominated by contributions from different coronal heights. Spectral imaging by the MWA, therefore, should make it possible to construct a three-dimensional distribution of electron density and temperature in the lower corona for the quiescent Sun.

\subsubsection{Electron acceleration and radio bursts}

Solar radio bursts are one of the primary remote signatures of electron acceleration in the corona and inner heliosphere. A wealth of information about bursts has been amassed, primarily from studies of their emission in frequency-time dynamic spectra. Their imaging studies remain rare. The standard models for these bursts involve the generation of radiation near the electron plasma frequency, $v_{\mathrm{pe}}$, as well as near $2 v_{\text {pe }}$, where

$$
v_{\mathrm{pe}} \approx 9 n_{\mathrm{e}}^{1 / 2} \mathrm{MHz}
$$

for electron number density, $n_{\mathrm{e}}$, in $\mathrm{m}^{-3}$. This mechanism involves the generation of electrostatic Langmuir waves and their conversion into radio emission via various linear and nonlinear processes (Ginzburg \& Zhelezniakov 1958; Melrose 1980; Robinson \& Cairns 2000; Cairns 2011). At MWA frequencies, beams of energetic electrons typically move away from the Sun; hence, emission frequency is expected to decrease with time since the coronal electron density decreases with radius.

We will focus on type II and III bursts with the MWA. Type II bursts are associated with suprathermal electrons accelerated at magnetohydrodynamic shocks to speeds of 400-2000 $\mathrm{km} \mathrm{s}^{-1}$, whereas type III bursts are due to bursts of electrons moving at speeds of the order of one-third of the speed of light, produced in magnetic reconnection sites during solar flares (Wild et al. 1963; Melrose 1980; Nelson \& Melrose 1985; Bastian et al. 1998; Robinson \& Cairns 2000; Cairns 2011).

Several major unresolved questions exist. The first is whether type II bursts are associated with a blast wave (i.e. a freely propagating shock) or the bow wave found upstream of a CME that is expanding into the heliosphere (Cane \& Erickson 2005; Vršnak \& Cliver 2008; Cairns 2011). The second problem is that estimates of density profiles that are derived by applying the plasma frequency relation to time-dependent spectra (Cairns et al. 2009; Lobzin et al. 2010) have tended to be much shallower than expected, as shown in Figure 10. The density profile is coupled to the formation mechanism of the corona; hence, discrepancy with models casts doubt on the 


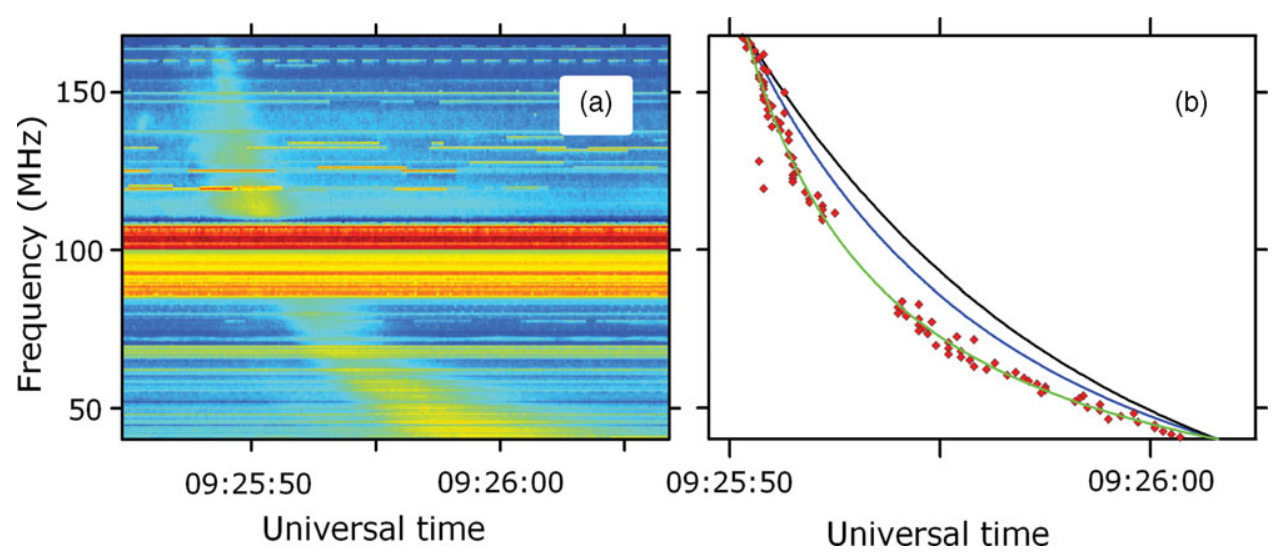

Figure 10. (a) Dynamic spectrum of the type III burst of 09:26 UTC on 1999 October 20, observed by the Potsdam-Tremsdorf Radiospectrograph, with normalised intensity (colour coded) as a function of frequency and time (Cairns et al. 2009). Horizontal bands are due to RFI. (b) Red diamonds show the $(f, t)$ locations of flux maxima for each time. The green line is the best fit to $v(t)=a(t-b)^{-\beta / 2}$ and implies that $n_{\mathrm{e}}(r) \sim\left(r-1.0 R_{\mathrm{S}}\right)^{-\beta}$ with $\beta=2.0 \pm 0.3$. This unexpected result is a solar wind-like profile for $n_{\mathrm{e}}(r)$. Fits for the leading power-law term $\beta=6$ of the standard density model based on coronagraph data (blue line) and an exponential gravitational-settling profile (black line) agree poorly with the type III data.

source of energetic electrons. Lastly, unresolved theoretical issues exist due to the relatively small levels of circular polarisation observed in type II and III bursts, despite existing theories predicting essentially $100 \%$ circular polarisation in the sense of the o mode (left hand) (Nelson \& Melrose 1985; Suzuki \& Dulk 1985; Cairns 2011).

The rapid spectroscopic imaging capability of MWA will address these issues by localising burst emission. An initial example of this capability is shown in Figure 11, in which the MWA development system imaged a solar burst at 15arcmin angular resolution (Oberoi et al. 2011). The 2-arcmin angular resolution of the full MWA will be nearly an order of magnitude better. This will help to determine the shock mechanism by providing radio positions to compare with CME observations (e.g. from STEREO), and it will improve density profile estimates by eliminating the need to infer burst location based on plasma frequency. In combination with the recent detailed models for the source regions and dynamics spectra of type II burst emission, as reviewed by Cairns (2011), the MWA images and dynamic spectra should help to determine definitively the relationship between type II bursts, CMEs, and shocks and to provide strong tests of our understanding of the propagation of events between the source and Earth.

\subsubsection{Direct imaging of emission from CME plasma}

Though CMEs are routinely imaged in visible light using coronagraphs, and CME-driven shocks produce broadband radio emissions over several hundred $\mathrm{MHz}$ to as narrow as $20 \mathrm{kHz}$, the CME plasma itself has been difficult to observe directly. Metre and decimetre frequencies are regarded as the most suitable in which to try to image the synchrotron, gyrosynchrotron, and/or thermal bremsstrahlung emission from the CME plasma (Bastian \& Gary 1997). Such measurements offer very useful and unique diagnostic capabilities, includ- ing the ability to constrain the CME magnetic field remotely. To date, however, only a few events have been confidently interpreted this way (e.g. Bastian et al. 2001; Maia et al. 2000). Observing these events is particularly challenging for existing instruments due to the large contrasts between the inherent brightness of the non-thermal emission from the CME plasma (weak) and solar disc (strong), and other phenomenon which are often simultaneously present (e.g. noise storms), and the large angular scales of this relatively weak emission. With its significantly higher dynamic range imaging ability and higher sensitivity to extended emission scales, owing to its numerous short baselines, the MWA should be the most suitable instrument yet for direct imaging of emissions from the CME plasma.

\subsection{Heliospheric science}

With an increasing reliance on the robust functioning of satellites, humankind is now more affected by space weather than ever before. Reliable predictions of space weather are needed to protect our technological assets in orbit as well as humans in space. The largest of the space weather events are caused by CMEs, and the geo-effectiveness of CMEs is closely tied to the presence of a southward-directed magnetic field in the CME or behind the CME-driven shock. Currently, the only operational means of obtaining this information is from the in situ observations by the near-Earth satellites. This approach suffers from two significant disadvantages: (1) These measurements provide a lead time of only a few hours; consequently, the advance warning comes so close to the event itself that it leaves little opportunity to take any remedial measures. (2) At these crucial times, interactions between the CME's magnetic field and plasma significantly alter the coupling between Earth's ionosphere and magnetosphere and the solar wind, leading to major changes in energetic particle 

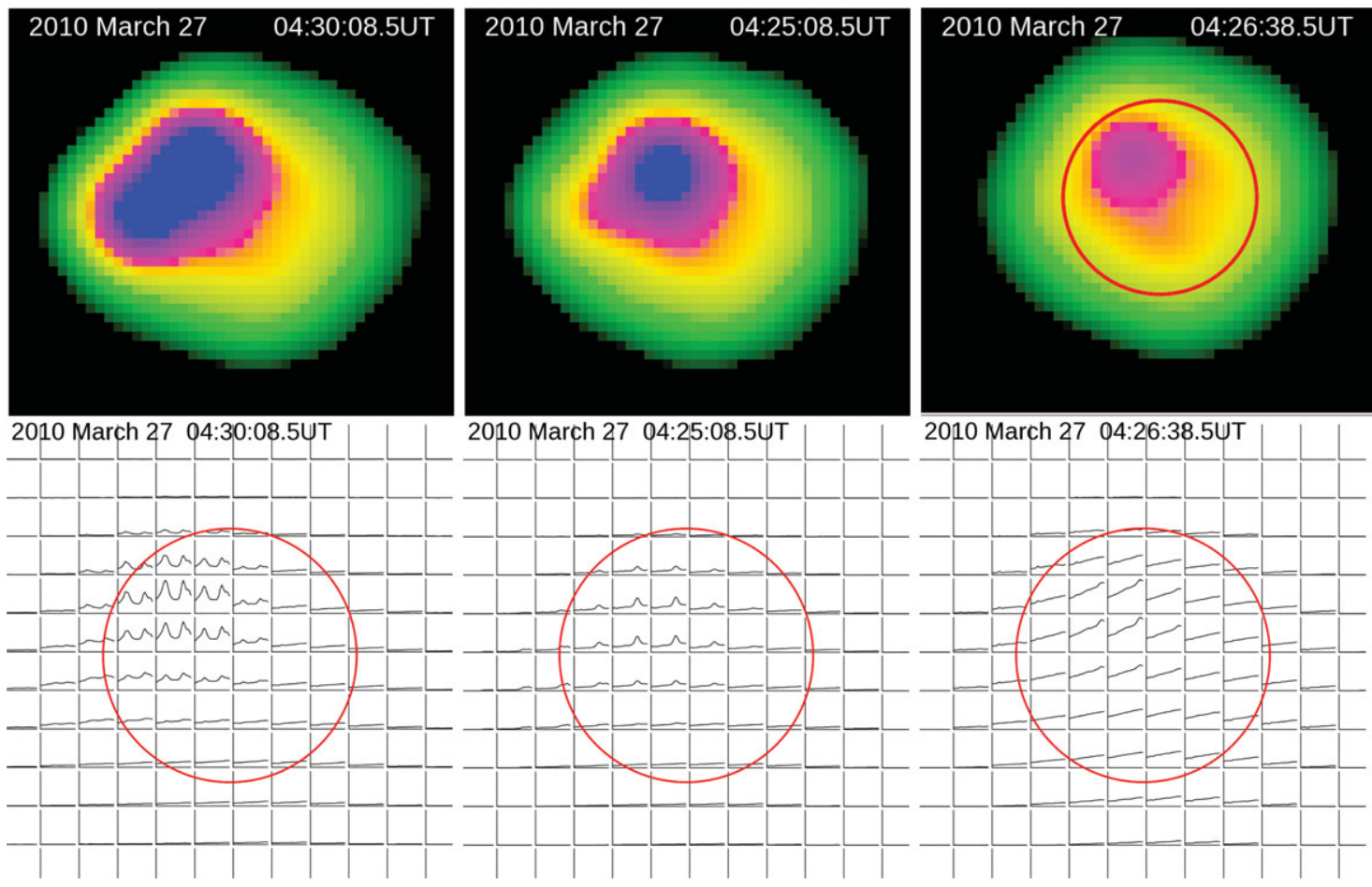

2010 March 27 04:26:38.5UT

Figure 11. The top panels show a set of radio images at $193.3 \mathrm{MHz}$ from the MWA development system on the same intensity scale (Oberoi et al. 2011). From left to right, the first one comes from a time close to the peak of a weak non-thermal emission feature lasting $\sim 10 \mathrm{~s}$ and occupying the entire $\sim 30 \mathrm{MHz}$ of observing bandwidth, the second is during one of numerous weaker events that spanned $\sim 5 \mathrm{~s}$ and $\sim 5 \mathrm{MHz}$, and the last one shows an image of steady solar emission for the nearly quiescent Sun, although with an active region still visible in the northern hemisphere. The dynamic range of these images is $\sim 2500$ and exceeds that of earlier images by about an order of magnitude. The bottom panels show spatially localised spectra of emission across the solar disc at the same times as the top panels. The spectra are shown for every third pixel, the pixel size is $100 \times 100 \operatorname{arcsec}^{2}$. The spectra span the entire observing bandwidth, binned into 24 frequency bands spaced $1.28 \mathrm{MHz}$ apart and averaged over $0.8 \mathrm{MHz}$. The $y$-axis ranges in arbitrary units are (left to right) 50-4 620,100-6000 and 100-1 800. Celestial north is on top, and the red circles mark the size of the optical solar disc.

flux, plasma density, plasma motion, magnetic and electric fields, and ionospheric activity. Degradation in satellite hardware and communication performance then occur, as well as many other space weather effects.

\subsubsection{Faraday rotation}

The observation of FR of background polarised radio sources (see also Section 3.3) is the only known remote sensing technique sensitive to the orientation and magnitude of the interplanetary magnetic field. Changes in FR associated with the passage of the solar corona or CMEs through the line of sight to spacecraft were convincingly detected using the telemetry signal from the Pioneer 6 and Helios satellites (Stelzried et al. 1970; Bird 1982; Bird et al. 1985). More recently, coronal FR has been detected using VLA observations of radio galaxies as the Sun passes in front of them (Mancuso \& Spangler 2000; Spangler \& Whiting 2009). The ability to routinely monitor and track CME magnetic fields would revolutionise the field of space weather.

FR measurements have been applied to many interesting studies of the nature of the solar corona and the solar wind, including to determine the mean magnetic field in the corona (Patzold et al. 1987), to measure the power spectrum of coro- nal waves and turbulence as a function of distance (Efimov et al. 1996), to suggest the existence of intermittent waves at single locations (Chashei et al. 1999), to support theoretical models of wave dissipation as a coronal heating mechanism (Hollweg et al. 1982), and to study the structure of the heliospheric current sheet (Mancuso \& Spangler 2000).

Studies so far have been severely limited by the paucity of suitable linearly polarised sources and the narrow fields of view of existing instrumentation (Spangler \& Whiting 2009). As discussed in Section 3.3, observations using the WSRT at 340-370 MHz have lead to detection of polarised extragalactic sources exhibiting typical polarised intensities of $20 \mathrm{mJy}$ and readily measurable RMs (Wieringa et al. 1993; Haverkorn, Katgert, \& de Bruyn 2003a, 2003b, 2003c). In the few areas of the sky investigated in detail, these efforts reveal about one suitable polarised source every $\sim 4 \mathrm{deg}^{2}$, as well as patches of diffuse polarised emission (Bernardi et al. 2013), suggesting that there will be sufficiently numerous background sources to chart CMEs.

The combination of the sensitivity of the MWA, its low frequency of operation, and its wide field of view, along with the availability of a large number of polarised sources with sufficient flux density, present the possibility of being able 
to measure the coronal and heliospheric FR simultaneously to several different lines of sight and out to much larger distances from the Sun ( $\sim 50$ solar radii) than previously accessible. No measurements of FR due to CME plasma are available yet at large solar offsets; hence, we rely on simulations to build quantitative expectations. Jensen et al. (2010) estimate the RM from CMEs to be $\sim 0.05 \mathrm{rad} \mathrm{m}^{-2}$ $\left(12^{\circ}\right.$ at $150 \mathrm{MHz}$ ) at about $0.4 \mathrm{AU}$. Liu et al. (2007) also arrive at a similar estimate and also show that the FR time series from instruments like the MWA during the CME passage may be used to infer the helicity and size of the CME.

For multiple reasons, these are rather challenging measurements to make (Oberoi \& Lonsdale 2013). Ionospheric $\mathrm{RM}$, is $1-10 \mathrm{rad} \mathrm{m}^{-2}$, more than an order of magnitude larger than the expected heliospheric or CME RM signature. This implies that we will need to achieve ionospheric RM calibration accuracies of a few per cent over large fields of view in order to study heliospheric RM. The quiet solar flux density is $10^{4}-10^{5} \mathrm{Jy}$; hence, detecting $20 \mathrm{mJy}$ sources requires polarimetric imaging dynamic range of about $10^{6}$, in the vicinity of a strong and variable Sun.

\subsubsection{Interplanetary scintillation}

As radio waves from a distant compact radio source propagate through the turbulent and inhomogeneous solar wind plasma, the incident plane wavefronts pick up phase corrugations corresponding to density inhomogeneities. These phase corrugations develop into intensity fluctuations by the time the wavefronts arrive at the Earth-based telescope. Due to the motion of the solar wind, the interference pattern produced by these intensity fluctuations is swept past the telescope giving rise to observed scintillations.

The evolution and dynamics of solar wind in the inner heliosphere dominates space weather effects. However, there are few techniques available to study the solar wind in this vast region. Historically, interplanetary scintillation (IPS) was the first remote sensing technique used to investigate the solar wind in this region (Hewish, Scott, \& Wills 1964). More recently, the routine availability of observations from heliospheric imagers (e.g. STEREO) that image very large parts of the heliosphere using white light scattered from the solar wind electrons, have emerged as very useful tools for investigations in the inner heliosphere. These techniques, along with local in situ sampling provided by most satellitebased instrumentation, complement each other well since they cover different spatial scales and have led to a more complete characterisation of the solar wind than would have been possible with either of the data sets (e.g. Jackson et al. 2007; Dorrian et al. 2008).

Several new instruments are coming online to study IPS. Apart from the MWA, the new facilities include the LOFAR (Bisi et al. 2011; Fallows et al. 2012) and the Mexico Array Telescope (Mejia-Ambriz et al. 2010). In addition, the Ooty Radio Telescope in India (P. K. Manoharan, private communication, 2012) and Solar Wind Imaging Facility (SWIFT) in Japan (Tokumaru et al. 2011) are both undergoing con- siderable upgrades which will substantially improve their scientific capabilities.

For the MWA, the primary IPS observable will be the power spectrum of observed intensity fluctuations, although we will also employ a less commonly used observable, the normalised co-spectrum obtained from simultaneous observations at frequencies differing by a factor of $\sim 2$ (Scott, Rickett, \& Armstrong 1983; Zhang 2007). Under the assumption of weak scattering, the interplanetary medium can be regarded as a collection of thin screens placed along the line of sight, and the IPS observable can be modelled as a weighted sum of the contributions from each of the thin screens. The physical parameters to which IPS is sensitive are (1) the velocity of the solar wind; (2) the level of electron density fluctuations, $\delta n_{\mathrm{e}}$; (3) the spectral index of the $\delta n_{\mathrm{e}}$ power spectrum; (4) the inner scale for the power law governing $\delta n_{\mathrm{e}}$; and (5) the axial ratio of $\delta n_{\mathrm{e}}$.

Using an inversion process, it is possible to extract the global three-dimensional distribution of IPM parameters (Jackson et al. 1998; Kojima et al. 1998; Asai et al. 1998; Oberoi 2000; Bisi et al. 2008, 2010; Dorrian et al. 2010; Xiong et al. 2011). The most important bottleneck currently constraining the fidelity and resolution of the inversion of IPS data, and hence its science output, are the sparseness (both in space and time) and the non-uniformity of the sampling of the heliosphere afforded by the present generation instrumentation (Oberoi \& Benkevitch 2010). The MWA will represent a significant advance in addressing the bottleneck of limited heliospheric sampling when its voltage capture system is completed. The MWA will have a sensitivity of $\sim 70 \mathrm{mJy}$ (1 s and $30.72 \mathrm{MHz}$ ), comparable to that of the SWIFT transit facility currently under commissioning in Japan. The MWA will boost the number of independent IPS measurements by up to a factor of $\sim 30$ over what is possible with most present-day systems. The southern hemisphere location of the MWA also complements the heliospheric coverage provided by the existing facilities, all of which happen to be in the northern hemisphere. Combining these data with other MWA observations like solar imaging and FR observations, and the observations from other sources such as STEREO, $S D O$, and HINODE, will provide a very comprehensive data set for characterising the inner heliosphere, monitoring and tracking CME evolution from close to the Sun to our terrestrial neighbourhood. Furthermore, the software framework being developed for handling the MWA IPS data could provide a common data repository, interface, and analysis tools for IPS data from observatories across the world (Oberoi \& Benkevitch 2010).

\subsection{Ionospheric science}

Propagation through the dynamic and structured magnetoionic ionospheric plasma leaves an imprint on the incident low radio frequency radiation from astronomical sources. In order to arrive at the true properties of the incident radiation, the ionospheric distortion must be identified and removed. 
The MWA can tackle this long-standing challenge by measuring the refractive shift in the apparent position of dozens of bright and relatively compact sources at a cadence of $8 \mathrm{~s}$. The ionospheric calibration information thus comprises of a time series of vectors showing the refractive shift of calibrator sources from their fiducial positions. To first order, the magnitude of the shift is directly proportional to the ionospheric gradient in the total electron content (TEC) towards the fiducial sky position of the calibrator source. This information for all the observed calibrator sources leads to a series of differential TEC maps and an accuracy of $\sim 0.01$ TECU $\left(1 \mathrm{TECU}=10^{16}\right.$ electrons $\left.\mathrm{m}^{-2}\right)$. These maps, produced routinely as a part of the MWA calibration, will form the primary data products for ionospheric science. The MWA beam will sample a region about $175 \mathrm{~km} \times 175 \mathrm{~km}$ at a height of $\sim 300$ $\mathrm{km}$ and the expected calibrator source density corresponds to spatial scales of $\sim 10 \mathrm{~km}$ at this ionospheric height.

The high-precision and dense sampling of the ionosphere provided by the MWA offers new opportunities for ionospheric science. The three areas of ionospheric research that the MWA will make significant contributions are in (1) the measuring of waves in TEC associated with travelling ionospheric disturbances (TIDs); (2) the development of storm enhanced density (SED) and sharp TEC gradients associated with space weather events; and (3) the tracking of the onset and evolution of scintillation associated with the development of ionospheric irregularities.

\subsubsection{Travelling ionospheric disturbances}

TIDs are manifestations of middle-scale ionospheric irregularities arising as response to acoustic gravity waves. TIDs are frequently observed at high and middle latitudes (Bristow, Greenwald, \& Villain 1996; Nicolls \& Heinselman 2007; Oliver et al. 1994, 1997; Djuth et al. 2004), and their activity and amplitudes vary depending on latitude, longitude, local time, season, and solar cycle (e.g. Kotake et al. 2006; Hernández-Pajares, Juan, \& Sanz 2006). They can be generated by auroral sources at the high latitude thermosphere, the passage of the solar terminator (Galushko et al. 2003), and storms, hurricanes, and tornados in the troposphere. Detailed information about the sources, energetics, and scale sizes of energy coupling from the lower atmosphere to the upper atmosphere, along with resulting ionospheric effects, is critical to developing a deep understanding of overall upper atmospheric energy balance. Measurements of TIDs at the VLA have been made since the early 1990s (Jacobson \& Erickson 1993; Jacobson et al. 1995) and recent observations have been reported by Helmboldt et al. (2012) and Helmboldt, Lane, \& Cotton (2012). The fine grid over which the MWA calibration solutions will provide differential TEC measurements will provide detailed measurements of the propagation of TIDs over the array and TEC gradients or irregularities.

\subsubsection{Storm enhanced density}

The MWA is well sited to contribute to coordinate international investigations of the SED phenomenon (Foster 1993).
This process is responsible for some of the largest space weather effects at mid-latitudes (Coster \& Foster 2007) since it produces severe, temporally dependent electron density gradients. These gradients will be obvious in the differential TEC measurements made by the MWA. The geographic/geomagnetic pole offset in the southern hemisphere is anticipated to influence the development of SED near the MWA (Coster et al. 2007; Foster \& Coster 2007; Foster \& Erickson 2013). SED associated gradients have a practical consequence, as they cause large errors in GPS range measurements, and significantly degrade the high-precision positioning information provided by GPS systems. The MWA's longitude $\left(\sim 116^{\circ} \mathrm{E}\right)$ is very close to the longitude being extensively instrumented in the Asian sector as part of the Chinese Meridian project $\left(\sim 113^{\circ}\right)$, and the conjugate longitude of $\sim 64^{\circ} \mathrm{W}$ is within the field of view of the US-sponsored chain of incoherent scatter radars, in particular Millstone Hill. The combination of simultaneous measurements from these distributed sensors thus provides a unique way to determine mesoscale response to geomagnetic disturbances in the coupled geospace system. In particular, it will be possible to investigate whether there is a seasonal and universal time dependence of geomagnetic storm response by comparing American and Asian/Australian sector response to solar wind and CME forcing. Such effects have been predicted theoretically (Foster \& Erickson 2013) but have not yet been confirmed by observations.

\subsubsection{Ionospheric scintillation}

Ionospheric scintillation can be defined as the rapid fluctuation of radio-frequency signal phase and/or amplitude, generated as a signal passes through the ionosphere. MWA observations using voltage capture capabilities will be uniquely suited for the exploration of ionospheric irregularities and scintillation. The voltage capture system should provide the ability to create array beams in post-processing that use the entire bandwidth and collecting area of the MWA, and that can be pointed independently anywhere within the MWA field of view. Such a system will provide scintillation measurements at multiple positions across the visible ionospheric region.

MWA array-beam observations will be augmented by data from specially designed GPS receivers at and near the MWA site that can measure $L$-band scintillation statistics. GPS TEC data from these receivers, when combined with data from a large number of newly deployed GPS receivers on the Australian continent, will provide important contextual information about the regional distribution of TEC around the MWA site. Other satellite-based remote-sensing platforms, including COSMIC, DMSP, and JASON, will also be available during overflights to provide further information about background ionospheric plasma and electrodynamic conditions in the ionosphere. A similar approach has been followed previously in the Combined Radio Interferometry and COSMIC Experiment in Tomography (CRICKET) campaign utilising the VLA (Dymond et al. 2008; Coker et al. 2009). This mix 
of data types allows for ionospheric specification from the global scale down to the regional scale.

\section{CONCLUSION}

The MWA is a unique low-frequency instrument with high survey efficiency and arcminute angular resolution. It will provide deep integrations on EoR target regions that could enable the first detection of the redshifted $21-\mathrm{cm}$ signal and detailed characterisation of diffuse Galactic emission and faint extragalactic source populations. The planned full-Stokes southern hemisphere sky survey will include the Galactic Centre, Galactic plane, and the Large and Small Magellanic Clouds, making the MWA particularly well suited to identifying the missing population of SNRs, and thereby constraining the total energy budget of the Galaxy.

Long integrations in the EoR fields and regular observations along the Galactic plane will enable sensitive searches for pulsars and transient events on timescales from seconds to days. Blind surveys for both rare and faint events are planned, as well as targeted observations of known transient sources, including X-ray binaries, neutron stars, pulsars, and low-mass stellar objects.

The MWA will open new windows into space weather and solar physics and provide new measurements of solar bursts throughout their journey from the surface of the Sun to the Earth and on to the outer Solar System. High-dynamic range spectroscopic imaging will show the origin of bursts in the solar corona, while monitoring of interplanetary scintillation will probe the interstellar wind. At Earth, the MWA's calibration system will yield detailed measurements of ionospheric disturbances travelling over the telescope.

The telescope is complementary to other new lowfrequency radio telescopes, falling between LOFAR and PAPER in both sensitivity and angular resolution. It is the only low-frequency SKA precursor and is located on the very radio-quiet site planned for the SKA low-band telescope. The combination of ASKAP and MWA at the MRO will yield a comprehensive programme of precursor science for the SKA over the coming years. The MWA possesses a versatile design with excess infrastructure capability that will allow the telescope and its users to embrace new scientific and technological opportunities over the coming decade, hopefully providing an enduring and fruitful future for the instrument.

\section{ACKNOWLEDGMENTS}

This scientific work makes use of the Murchison Radio-astronomy Observatory. We acknowledge the Wajarri Yamatji people as the traditional owners of the Observatory site. Support for the MWA comes from the U.S. National Science Foundation (grants AST0457585, PHY-0835713, CAREER-0847753, and AST-0908884), the Australian Research Council (LIEF grants LE0775621 and LE0882938), the U.S. Air Force Office of Scientific Research (grant FA9550-0510247), and the Centre for All-sky Astrophysics (an Australian Research Council Centre of Excellence funded by grant
CE110001020). Support is also provided by the Smithsonian Astrophysical Observatory, the MIT School of Science, the Raman Research Institute, the Australian National University, and the Victoria University of Wellington (via grant MED-E1799 from the New Zealand Ministry of Economic Development and an IBM Shared University Research Grant). The Australian Federal government provides additional support via the National Collaborative Research Infrastructure Strategy, Education Investment Fund, and the Australia India Strategic Research Fund, and Astronomy Australia Limited, under contract to Curtin University. We acknowledge the iVEC Petabyte Data Store, the Initiative in Innovative Computing and the CUDA Center for Excellence sponsored by NVIDIA at Harvard University, and the International Centre for Radio Astronomy Research (ICRAR), a Joint Venture of Curtin University and The University of Western Australia, funded by the Western Australian State government.

\section{REFERENCES}

Alves, M. I. R., Davies, R. D., Dickinson, C., Calabretta, M., Davis, R., \& Staveley-Smith, L. 2012, MNRAS, 422, 2429

Amy, S. W., Large, M. I., \& Vaughan, A. E. 1989, PASAu, 8, 172 Antonova, A., Doyle, J. G., Hallinan, G., Golden, A., \& Koen, C. 2007, A\&A, 472, 257

Araya-Melo, P. A., Aragón-Calvo, M. A., Brüggen, M., \& Hoeft, M. 2012, MNRAS, 423, 2325

Asai, K., Kojima, M., Tokumaru, M., Yokobe, A., Jackson, B. V., Hick, P. L., \& Manoharan, P. K. 1998, J. Geophys. Res., 103, 1991

Asgekar, A., \& Deshpande, A. A. 2001, MNRAS, 326, 1249

Audard, M., Osten, R. A., Brown, A., Briggs, K. R., Güdel, M., Hodges-Kluck, E., \& Gizis, J. E. 2007, A\&A, 471, L63

Baird, G. A., Meikle, W. P. S., Jelley, J. V., Palumbo, G. G. C., \& Partridge, R. B. 1976, Ap\&SS, 42, 69

Balsano, R. J. 1999, PhD thesis, Princeton University

Banfield, J. K., George, S. J., Taylor, A. R., Stil, J. M., Kothes, R., \& Scott, D. 2011, ApJ, 733, 69

Bania, T. M., Anderson, L. D., Balser, D. S., \& Rood, R. T. 2010, ApJ, 718, L106

Bannister, K. W., Murphy, T., Gaensler, B. M., Hunstead, R. W., \& Chatterjee, S. 2011, MNRAS, 412, 634

Barkana, R., \& Loeb, A. 2005, ApJ, 624, L65

Barkana, R., \& Loeb, A. 2008, MNRAS, 384, 1069

Bastian, T. S. 2004, P\&SS, 52, 1381

Bastian, T. S., Benz, A. O., \& Gary, D. E. 1998, ARA\&A, 36, 131

Bastian, T. S., Dulk, G. A., \& Leblanc, Y. 2000, ApJ, 545, 1058

Bastian, T. S., \& Gary, D. E. 1997, J. Geophys. Res., 102, 14031

Bastian, T. S., Pick, M., Kerdraon, A., Maia, D., \& Vourlidas, A. 2001, ApJ, 558, L65

Beardsley, A. P., et al. 2013, MNRAS, 429, L5

Beck, R., \& Gaensler, B. M. 2004, NewAR, 48, 1289

Becker, G. D., Rauch, M., \& Sargent, W. L. W. 2007, ApJ, 662, 72

Belikov, A. V., \& Hooper, D. 2009, PhRvD, 80, 035007

Benz, A. O., \& Paesold, G. 1998, A\&A, 329, 61

Berger, E. 2006, ApJ, 648, 629

Berger, E., et al. 2001, Nature, 410, 338

Bernardi, G., Mitchell, D. A., Ord, S. M., Greenhill, L. J., Pindor, B., Wayth, R. B., \& Wyithe, J. S. B. 2011, MNRAS, 413, 411

Bernardi, G., et al. 2009, A\&A, 500, 965

Bernardi, G., et al. 2013, ApJ, submitted 
Bhat, N. D. R., Cordes, J. M., Camilo, F., Nice, D. J., \& Lorimer, D. R. 2004, ApJ, 605, 759

Bhat, N. D. R., et al. 2007, ApJ, 665, 618

Bird, M. K. 1982, SSRv, 33, 99

Bird, M. K., et al. 1985, SoPh, 98, 341

Bisi, M. M., Fallows, R. A., Jensen, E. A., Breen, A., Xiong, M., \& Jackson, B. V. 2011, AGU Fall Meeting Abstracts, C2020

Bisi, M. M., Jackson, B. V., Breen, A. R., Dorrian, G. D., Fallows, R. A., Clover, J. M., \& Hick, P. P. 2010, SoPh, 265, 233

Bisi, M. M., Jackson, B. V., Hick, P. P., Buffington, A., Odstrcil, D., \& Clover, J. M. 2008, J. Geophys. Res. (Space Phys.), 113, 0

Blasi, P., \& Colafrancesco, S. 1999, APh, 12, 169

Bower, G. C., Roberts, D. A., Yusef-Zadeh, F., Backer, D. C. Cotton, W. D., Goss, W. M., Lang, C. C., \& Lithwick, Y. 2005 , ApJ, 633, 218

Bower, G. C., Saul, D., Bloom, J. S., Bolatto, A., Filippenko, A. V., Foley, R. J., \& Perley, D. 2007, ApJ, 666, 346

Bowman, J. D., Morales, M. F., \& Hewitt, J. N. 2006, ApJ, 638, 20

Bowman, J. D., Morales, M. F., \& Hewitt, J. N. 2009, ApJ, 695, 183

Boyles, J., et al. 2013, ApJ, 763, 80

Brentjens, M. A., \& de Bruyn, A. G. 2005, A\&A, 441, 1217

Bristow, W. A., Greenwald, R. A., \& Villain, J. P. 1996, J. Geophys. Res., 101, 15685

Brogan, C. L., Gelfand, J. D., Gaensler, B. M., Kassim, N. E., \& Lazio, T. J. W. 2006, ApJ, 639, L25

Brown, S. D. 2011, JApA, 32, 577

Brown, S., Farnsworth, D., \& Rudnick, L. 2010, MNRAS, 402, 2

Brunetti, G., Setti, G., Feretti, L., \& Giovannini, G. 2001, MNRAS, 320,365

Burgasser, A. J., \& Putman, M. E. 2005, ApJ, 626, 486

Burke-Spolaor, S., \& Bailes, M. 2010, MNRAS, 402, 855

Burkhart, B., Lazarian, A., \& Gaensler, B. M. 2012, ApJ, 749, 145

Cairns, I. H. 2004, P\&SS, 52, 1423

Cairns, I. H. 2011, in Coherent Radio Emissions Associated with Star System Shocks, ed. M. P. Miralles \& J. Sánchez Almeida (Berlin: Springer), 267

Cairns, I. H., Lobzin, V. V., Warmuth, A., Li, B., Robinson, P. A., \& Mann, G. 2009, ApJ, 706, L265

Cameron, P. B., et al. 2005, Nature, 434, 1112

Camilo, F., Ransom, S. M., Halpern, J. P., \& Reynolds, J. 2007, ApJ, 666, L93

Camilo, F., Ransom, S. M., Halpern, J. P., Reynolds, J., Helfand, D. J., Zimmerman, N., \& Sarkissian, J. 2006, Nature, 442, 892

Cane, H. V., \& Erickson, W. C. 2005, ApJ, 623, 1180

Cane, H. V., Erickson, W. C., \& Prestage, N. P. 2002, J. Geophys. Res. (Space Phys.), 107, 1315

Cassano, R., Brunetti, G., Norris, R. P., Röttgering, H. J. A., Johnston-Hollitt, M., \& Trasatti, M. 2012, A\&A, 548, A100

Cassano, R., Brunetti, G., Röttgering, H. J. A., \& Brüggen, M. 2010, A\&A, 509, A68

Cen, R., \& Ostriker, J. P. 1999, ApJ, 514, 1

Chakraborti, S., Ray, A., Soderberg, A. M., Loeb, A., \& Chandra, P. 2011, Nature Commun., 2, 175

Charbonneau, D., Brown, T. M., Noyes, R. W., \& Gilliland, R. L. 2002, ApJ, 568, 377

Chashei, I. V., Bird, M. K., Efimov, A. I., Andreev, V. E., \& Samoznaev, L. N. 1999, SoPh, 189, 399

Chen, X., \& Miralda-Escudé, J. 2004, ApJ, 602, 1

Coker, C., Thonnard, S. E., Dymond, K. F., Lazio, T. J. W., Makela, J. J., \& Loughmiller, P. J. 2009, Radio Sci., 44, 0
Colgate, S. A., \& Noerdlinger, P. D. 1971, ApJ, 165, 509

Condon, J. J. 1974, ApJ, 188, 279

Condon, J. J., Cotton, W. D., Greisen, E. W., Yin, Q. F., Perley, R. A., Taylor, G. B., \& Broderick, J. J. 1998, AJ, 115, 1693

Corbel, S., Kaaret, P., Fender, R. P., Tzioumis, A. K., Tomsick, J. A., \& Orosz, J. A. 2005, ApJ, 632, 504

Cordes, J. M., \& Lazio, T. J. W. 2002, astro-ph/0207156

Cordes, J. M., Lazio, T. J. W., \& McLaughlin, M. A. 2004, NewAR, 48,1459

Cordes, J. M., \& McLaughlin, M. A. 2003, ApJ, 596, 1142

Cordes, J. M., Weisberg, J. M., Frail, D. A., Spangler, S. R., \& Ryan, M. 1991, Nature, 354, 121

Coster, A. J., Colerico, M. J., Foster, J. C., Rideout, W., \& Rich, F. 2007, Geophys. Res. Lett., 34, L18105

Coster, A. J., \& Foster, J. C. 2007, Radio Sci. Bull., 321, 28

Croft, S., Bower, G. C., Keating, G., Law, C., Whysong, D., Williams, P. K. G., \& Wright, M. 2011, ApJ, 731, 34

Datta, A., Bowman, J. D., \& Carilli, C. L. 2010, ApJ, 724, 526

Datta, K. K., Majumdar, S., Bharadwaj, S., \& Choudhury, T. R. 2008, MNRAS, 391, 1900

de Bruyn, A. G., Katgert, P., Haverkorn, M., \& Schnitzeler, D. H. F. M. 2006, AN, 327, 487

de Oliveira-Costa, A., Tegmark, M., Gaensler, B. M., Jonas, J., Landecker, T. L., \& Reich, P. 2008, MNRAS, 388, 247

Dennison, B. 1980, ApJ, 239, L93

Deshpande, A. A., \& Rankin, J. M. 1999, ApJ, 524, 1008

Dessenne, C. A.-C., et al. 1996, MNRAS, 281, 977

Djuth, F. T., Sulzer, M. P., Gonzáles, S. A., Mathews, J. D., Elder, J. H., \& Walterscheid, R. L. 2004, Geophys. Res. Lett., 31, L16801

Donati, J.-F., Forveille, T., Cameron, A. C., Barnes, J. R., Delfosse, X., Jardine, M. M., \& Valenti, J. A. 2006, Sci, 311, 633

Dorrian, G. D., Breen, A. R., Brown, D. S., Davies, J. A., Fallows, R. A., \& Rouillard, A. P. 2008, Geophys. Res. Lett., 35,24104

Dorrian, G. D., et al. 2010, SoPh, 265, 207

Duncan, R. C., \& Thompson, C. 1992, ApJ, 392, L9

Dymond, K., et al. 2008, in COSPAR Meeting, Vol. 37, 37th COSPAR Scientific Assembly, Montréal, Canada, 775

Efimov, A. I., Bird, M. K., Andreev, V. E., \& Samoznaev, L. N. 1996, AstL, 22, 785

Eikenberry, S. S., Matthews, K., Muno, M., Blanco, P. R., Morgan, E. H., \& Remillard, R. A. 2000, ApJ, 532, L33

Erickson, W. C., McConnell, D., \& Anantharamaiah, K. R. 1995, ApJ, 454, 125

Fallows, R. A., Asgekar, A., Bisi, M. M., Breen, A. R., \& ter-Veen, S. 2012, SoPh, doi:10.1007/s11207-012-9989-5

Fan, X., et al. 2006, AJ, 132, 117

Farrell, W. M., Desch, M. D., \& Zarka, P. 1999, J. Geophys. Res., 104,14025

Fender, R. 2006, in Compact Stellar X-ray Sources, ed. W. Lewin \& M. van der Klis (Cambridge, UK: Cambridge University Press), 381

Fender, R. P., Belloni, T. M., \& Gallo, E. 2004, MNRAS, 355, 1105

Fender, R. P., et al. 2006, in Proc. VI Microquasar Workshop: Microquasars and Beyond, September 18-22, Como, Italy.

Ferrari, C., Govoni, F., Schindler, S., Bykov, A. M., \& Rephaeli, Y. 2008, SSRv, 134, 93

Field, G. 1958, Proc. IRE, 46, 240

Fitt, A. J., Alexander, P., \& Cox, M. J. 1988, MNRAS, 230, 907 
Fletcher, A., \& Shukurov, A. 2007, in EAS Publications Series, Vol. 23, ed. M.-A. Miville-Deschênes \& F. Boulanger (Cambridge University Press), 109

Foster, J. C. 1993, J. Geophys. Res., 98, 1675

Foster, J. C., \& Coster, A. J. 2007, J. Atmos. Sol.-Terr. Phys., 69, 1241

Foster, J. C., \& Erickson, P. J. 2013, J. Atmos. Sol.-Terr. Phys., submitted

Frail, D. A., Kulkarni, S. R., \& Bloom, J. S. 1999, Nature, 398, 127

Frail, D. A., Kulkarni, S. R., Ofek, E. O., Bower, G. C., \& Nakar, E. 2012, ApJ, 747, 70

Furlanetto, S. R., Oh, S. P., \& Briggs, F. H. 2006, PhR, 433, 181

Furlanetto, S. R., Zaldarriaga, M., \& Hernquist, L. 2004, ApJ, 613,1

Gaensler, B. M., Dickey, J. M., McClure-Griffiths, N. M., Green, A. J., Wieringa, M. H., \& Haynes, R. F. 2001, ApJ, 549, 959

Gaensler, B., Haverkorn, M., Staveley-Smith, L., Dickey, J., McClure-Griffiths, N., Dickel, J., \& Wolleben, M. 2005a, in The Magnetized Plasma in Galaxy Evolution, ed. K. T. Chyzy, K. Otmianowska-Mazur, M. Soida, \& R.-J. Dettmar (Kraków: Jagiellonian University), 209

Gaensler, B. M., et al. 2005b, Nature, 434, 1104

Gaensler, B. M., et al. 2011, Nature, 478, 214

Galushko, V. G., Beley, V. S., Koloskov, A. V., Yampolski, Y. M., Paznukhov, V. V., Reinisch, B. W., Foster, J. C., \& Erickson, P. 2003, Radio Sci., 38, 060000

Geil, P. M., Gaensler, B. M., \& Wyithe, J. S. B. 2011, MNRAS, 418, 516

Geil, P. M., Wyithe, J. S. B., Petrovic, N., \& Oh, S. P. 2008, MNRAS, 390, 1496

Ginzburg, V. L., \& Zhelezniakov, V. V. 1958, SvA, 2, 653

Green, D. A. 2004, BASI, 32, 335

Grießmeier, J.-M., Zarka, P., \& Spreeuw, H. 2007, A\&A, 475, 359

Güdel, M. 2002, ARA\&A, 40, 217

Güdel, M., \& Benz, A. O. 1993, ApJ, 405, L63

Gupta, Y., \& Gangadhara, R. T. 2003, ApJ, 584, 418

Gurnett, D. A. 1974, J. Geophys. Res., 79, 4227

Hales, S. E. G., Baldwin, J. E., \& Warner, P. J. 1988, MNRAS, 234 , 919

Hallinan, G., et al. 2007, ApJ, 663, L25

Halpern, J. P., Gotthelf, E. V., Becker, R. H., Helfand, D. J., \& White, R. L. 2005, ApJ, 632, L29

Hansen, B. M. S., \& Lyutikov, M. 2001, MNRAS, 322, 695

Hassall, T. E., et al. 2012, A\&A, 543, A66

Haverkorn, M., Gaensler, B. M., McClure-Griffiths, N. M., Dickey, J. M., \& Green, A. J. 2006, ApJS, 167, 230

Haverkorn, M., \& Heitsch, F. 2004, A\&A, 421, 1011

Haverkorn, M., Katgert, P., \& de Bruyn, A. G. 2003a, A\&A, 403, 1045

Haverkorn, M., Katgert, P., \& de Bruyn, A. G. 2003b, A\&A, 403, 1031

Haverkorn, M., Katgert, P., \& de Bruyn, A. G. 2003c, A\&A, 404, 233

Haynes, R. F., Murray, J. D., Klein, U., \& Wielebinski, R. 1986, A\&A, 159, 22

Helmboldt, J. F., Lane, W. M., \& Cotton, W. D. 2012, Radio Sci. 47, RS5008

Helmboldt, J. F., Lazio, T. J. W., Intema, H. T., \& Dymond, K. F. 2012, Radio Sci., 47, 0
Hernández-Pajares, M., Juan, J. M., \& Sanz, J. 2006, J. Geophys. Res. (Space Phys.), 111, A07S11

Hewish, A., Scott, P. F., \& Wills, D. 1964, Nature, 203, 1214

Hollitt, C., \& Johnston-Hollitt, M. 2012, PASA, 29, 309

Hollweg, J. V., Bird, M. K., Volland, H., Edenhofer, P., Stelzried, C. T., \& Seidel, B. L. 1982, J. Geophys. Res., 87, 1

Hughes, A., Wong, T., Ekers, R., Staveley-Smith, L., Filipovic, M., Maddison, S., Fukui, Y., \& Mizuno, N. 2006, MNRAS, 370, 363

Hyman, S. D., Lazio, T. J. W., Kassim, N. E., \& Bartleson, A. L. 2002, AJ, 123, 1497

Hyman, S. D., Lazio, T. J. W., Kassim, N. E., Ray, P. S., Markwardt, C. B., \& Yusef-Zadeh, F. 2005, Nature, 434, 50

Hyman, S. D., Wijnands, R., Lazio, T. J. W., Pal, S., Starling, R., Kassim, N. E., \& Ray, P. S. 2009, ApJ, 696, 280

Ichikawa, K., Barkana, R., Iliev, I. T., Mellema, G., \& Shapiro, P. R. 2010, MNRAS, 406, 2521

Inoue, S. 2004, MNRAS, 348, 999

Ishwara-Chandra, C. H., Rao, A. P., Pandey, M., Manchanda, R. K., \& Durouchoux, P. 2005, ChJAAS, 5, 87

Jackson, B. V., Hick, P. P., Buffington, A., Bisi, M. M., Kojima, M., \& Tokumaru, M. 2007, A\&AT, 26, 477

Jackson, B. V., Hick, P. L., Kojima, M., \& Yokobe, A. 1998, J. Geophys. Res., 103, 12049

Jacobson, A. R., Carlos, R. C., Massey, R. S., \& Wu, G. 1995, J. Geophys. Res., 100, 1653

Jacobson, A. R., \& Erickson, W. C. 1993, Ann. Geophys., 11, 869

Jaeger, T. R., Hyman, S. D., Kassim, N. E., \& Lazio, T. J. W. 2012, AJ, 143, 96

Jenet, F. A., Anderson, S. B., \& Prince, T. A. 2001, ApJ, 558, 302

Jensen, E. A., Hick, P. P., Bisi, M. M., Jackson, B. V., Clover, J., \& Mulligan, T. 2010, SoPh, 265, 31

Johns-Krull, C. M., \& Valenti, J. A. 1996, ApJ, 459, L95

Johnston, S. 2003, MNRAS, 340, L43

Johnston, S., Hobbs, G., Vigeland, S., Kramer, M., Weisberg, J. M., \& Lyne, A. G. 2005, MNRAS, 364, 1397

Kaiser, N. 1987, MNRAS, 227, 1

Kaplan, D. L. 2008, in AIP Conf. Ser. Vol. 968, Astrophysics of Compact Objects, ed. Y.-F. Yuan, X.-D. Li, \& D. Lai (Melville: AIP), 129

Kaplan, D. L., Hyman, S. D., Roy, S., Bandyopadhyay, R. M., Chakrabarty, D., Kassim, N. E., Lazio, T. J. W., \& Ray, P. S. 2008, ApJ, 687, 262

Kaplan, D. L., Kulkarni, S. R., Frail, D. A., \& van Kerkwijk, M. H. 2002, ApJ, 566, 378

Kaplan, D. L., Kulkarni, S. R., \& van Kerkwijk, M. H. 2003, ApJ, 588, L33

Kaplan, D. L., \& van Kerkwijk, M. H. 2009, ApJ, 705, 798

Karlický, M. 2003, SSRv, 107, 81

Katz, C. A., Hewitt, J. N., Corey, B. E., \& Moore, C. B. 2003, PASP, 115,675

Keane, E. F., \& Kramer, M. 2008, MNRAS, 391, 2009

Keane, E. F., Kramer, M., Lyne, A. G., Stappers, B. W., \& McLaughlin, M. A. 2011, MNRAS, 415, 3065

Keane, E. F., Ludovici, D. A., Eatough, R. P., Kramer, M., Lyne, A. G., McLaughlin, M. A., \& Stappers, B. W. 2010, MNRAS, 401, 1057

Keane, E. F., Stappers, B. W., Kramer, M., \& Lyne, A. G. 2012, MNRAS, 425, L71

Keith, M. J., et al. 2010, MNRAS, 409, 619

Keller, S. C., et al. 2007, PASA, 24, 1 
Kempner, J. C., Blanton, E. L., Clarke, T. E., Enßlin, T. A., Johnston-Hollitt, M., \& Rudnick, L. 2004, in The Riddle of Cooling Flows in Galaxies and Clusters of Galaxies, ed. T. Reiprich, J. Kempner, \& N. Soker, 335. Available at: http://www.astro.virginia.edu/coolflow/

Keshet, U., Waxman, E., \& Loeb, A. 2004, ApJ, 617, 281

Kojima, M., Tokumaru, M., Watanabe, H., Yokobe, A., Asai, K., Jackson, B. V., \& Hick, P. L. 1998, J. Geophys. Res., 103, 1981

Komatsu, E., et al. 2009, ApJS, 180, 330

Kondratiev, V. I., McLaughlin, M. A., Lorimer, D. R., Burgay, M., Possenti, A., Turolla, R., Popov, S. B., \& Zane, S. 2009, ApJ, 702, 692

Kondratiev, V., Stappers, B., \& the LOFAR Pulsar Working Group. 2012, in IAU Symposium 291: Neutron Stars and Pulsars: Challenges and Opportunities after 80 Years, ed. J. van Leeuwen, arXiv: 1210.7005

Kondratiev, V., \& the LOFAR Pulsar Working Group. 2012, in IAU Symposium 291: Neutron Stars and Pulsars: Challenges and Opportunities after 80 Years, ed. J. van Leeuwen, arXiv:1210.6994

Kotake, N., Otsuka, Y., Tsugawa, T., Ogawa, T., \& Saito, A. 2006, J. Geophys. Res. (Space Phys.), 111, A04306

Kramer, M., Lyne, A. G., O'Brien, J. T., Jordan, C. A., \& Lorimer, D. R. 2006, Sci, 312, 549

Kulkarni, S. R., \& Phinney, E. S. 2005, Nature, 434, 28

Kumar, S. S. 1962, AJ, 67, 579

Law, C. J., \& Bower, G. C. 2012, ApJ, 749, 143

Law, C. J., Bower, G. C., Pokorny, M., Rupen, M. P., \& Sowinski, K. 2012, ApJ, 760, 124

Lazio, T. J. W., \& Farrell, W. M. 2007, ApJ, 668, 1182

Lazio, T. J. W., Farrell, W. M., Dietrick, J., Greenlees, E., Hogan, E., Jones, C., \& Hennig, L. A. 2004, ApJ, 612, 511

Lenc, E., Garrett, M. A., Wucknitz, O., Anderson, J. M., \& Tingay, S. J. 2008, ApJ, 673, 78

Li, Z., Wheeler, J. C., Bash, F. N., \& Jefferys, W. H. 1991, ApJ, 378,93

Lidz, A., Zahn, O., Furlanetto, S. R., McQuinn, M., Hernquist, L., \& Zaldarriaga, M. 2009, ApJ, 690, 252

Lidz, A., Zahn, O., McQuinn, M., Zaldarriaga, M., \& Hernquist, L. 2008, ApJ, 680, 962

Liu, Y., Manchester, W. B., IV, Kasper J. C., Richardson, J. D., \& Belcher, J. W. 2007, ApJ, 665, 1439

Liu, A., \& Tegmark, M. 2011, PhRvD, 83, 103006

Liu, A., Tegmark, M., Bowman, J., Hewitt, J., \& Zaldarriaga, M. 2009a, MNRAS, 398, 401

Liu, A., Tegmark, M., \& Zaldarriaga, M. 2009b, MNRAS, 394, 1575

Lobzin, V. V., Cairns, I. H., Robinson, P. A., Warmuth, A., Mann, G., Gorgutsa, R. V., \& Fomichev, V. V. 2010, ApJ, 724, 1099

Lonsdale, C. J., et al. 2009, IEEE Proc., 97, 1497

Lorimer, D. R., Bailes, M., McLaughlin, M. A., Narkevic, D. J., \& Crawford, F. 2007, Sci, 318, 777

Lynch, R. S., et al. 2013, ApJ, 763, 81

Lyne, A. G., McLaughlin, M. A., Keane, E. F., Kramer, M., Espinoza, C. M., Stappers, B. W., Palliyaguru, N. T., \& Miller, J. 2009, MNRAS, 400, 1439

Maan, Y., et al. 2013, ApJS, 204, 12

Macquart, J.-P. 2007, ApJ, 658, L1

Maia, D., Pick, M., Vourlidas, A., \& Howard, R. 2000, ApJ, 528, L49

Mancuso, S., \& Spangler, S. R. 2000, ApJ, 539, 480
Mao, S. A., Gaensler, B. M., Stanimirović, S., Haverkorn, M., McClure-Griffiths, N. M., Staveley-Smith, L., \& Dickey, J. M. 2008, ApJ, 688, 1029

Marcy, G., Butler, R. P., Fischer, D., Vogt, S., Wright, J. T., Tinney, C. G., \& Jones, H. R. A. 2005, Prog. Theor. Phys. Suppl., 158,24

Maron, O., Kijak, J., Kramer, M., \& Wielebinski, R. 2000, A\&AS, 147, 195

Matsumura, N., et al. 2007, AJ, 133, 1441

Matsumura, N., et al. 2009, AJ, 138, 787

McClintock, J. E., \& Remillard, R. A. 2006, in Compact Stellar X-ray Sources, ed. W. Lewin \& M. vanderKlis (Cambridge, UK: Cambridge University Press), 157

McLaughlin, M. A., et al. 2006, Nature, 439, 817

McLaughlin, M. A., et al. 2007, ApJ, 670, 1307

McLean, D. J., \& Labrum, N. R. 1985, Solar Radiophysics: Studies of Emission from the Sun at Metre Wavelengths (Cambridge: Cambridge University Press)

McQuinn, M., Lidz, A., Zahn, O., Dutta, S., Hernquist, L., \& Zaldarriaga, M. 2007, MNRAS, 377, 1043

McQuinn, M., Zahn, O., Zaldarriaga, M., Hernquist, L., \& Furlanetto, S. R. 2006, ApJ, 653, 815

Mejia-Ambriz, J. C., Villanueva-Hernandez, P., GonzalezEsparza, J. A., Aguilar-Rodriguez, E., \& Jeyakumar, S. 2010, SoPh, 265, 309

Mellema, G., Iliev, I. T., Pen, U.-L., \& Shapiro, P. R. 2006, MNRAS, 372,679

Melrose, D. B. 1980, SSRv, 26, 3

Melrose, D. B. 2003, Plasma Phys. Control. Fusion, 45, 523

Mesa, D., Baccigalupi, C., De Zotti, G., Gregorini, L., Mack, K.-H., Vigotti, M., \& Klein, U. 2002, A\&A, 396, 463

Mesinger, A. 2010, MNRAS, 407, 1328

Mirabel, I. F., \& Rodríguez, L. F. 1999, ARA\&A, 37, 409

Mitchell, D. A., Greenhill, L. J., Wayth, R. B., Sault, R. J., Lonsdale, C. J., Cappallo, R. J., Morales, M. F., \& Ord, S. M. 2008, IEEE J. Sel. Top. Signal Process., 2, 707

Moortgat, J., \& Kuijpers, J. 2005, in 22nd Texas Symposium on Relativistic Astrophysics, Stanford University, Palo Alto, CA, USA, ed. P. Chen, E. Bloom, G. Madejski, \& V. Patrosian, 326

Morales, M. F., Bowman, J. D., \& Hewitt, J. N. 2006, ApJ, 648, 767

Morales, M. F., Hazelton, B., Sullivan, I., \& Beardsley, A. 2012, ApJ, 752, 137

Morales, M. F., \& Hewitt, J. 2004, ApJ, 615, 7

Morales, M. F., Hewitt, J. N., Kasper, J. C., Lane, B., Bowman, J., Ray, P. S., \& Cappallo, R. J. 2005, ASPC, 345, 512

Nakar, E., \& Piran, T. 2011, Nature, 478, 82

Nelson, G. J., \& Melrose, D. B. 1985, Type II Bursts, ed. D. J. McLean \& N. R. Labrum (Cambridge and New York, Cambridge University Press), 333

Nicolls, M. J., \& Heinselman, C. J. 2007, Geophys. Res. Lett., 34, L21104

Nijboer, R. J., Pandey-Pommier, M., \& de Bruyn, A. G. 2009, SKA Memo \#113, http://www.skatelescope.org/publications/

Nord, M. E., Henning, P. A., Rand, R. J., Lazio, T. J. W., \& Kassim, N. E. 2006, AJ, 132, 242

Noutsos, A., Johnston, S., Kramer, M., \& Karastergiou, A. 2008, MNRAS, 386, 1881

Oberoi, D. 2000, PhD thesis, Indian Institute of Science

Oberoi, D., \& Benkevitch, L. 2010, SoPh, 265, 293

Oberoi, D., \& Kasper, J. C. 2004, P\&SS, 52, 1415 
Oberoi, D., \& Lonsdale, C. J. 2013, Radio Sci. 47, RS0K08

Oberoi, D., et al. 2011, ApJ, 728, L27

Oliver, W. L., Fukao, S., Yamamoto, Y., Takami, T., Yamanaka, M. D., Yamamoto, M., Nakamura, T., \& Tsuda, T. 1994, J. Geophys. Res., 99, 6321

Oliver, W. L., Otsuka, Y., Sato, M., Takami, T., \& Fukao, S. 1997, J. Geophys. Res., 102, 14499

Ord, S. M., Johnston, S., \& Sarkissian, J. 2007, SoPh, 245, 109

Osten, R. A., \& Bastian, T. S. 2006, ApJ, 637, 1016

Osten, R. A., Hawley, S. L., Bastian, T. S., \& Reid, I. N. 2006, ApJ, 637, 518

Paladini, R., Burigana, C., Davies, R. D., Maino, D., Bersanelli, M., Cappellini, B., Platania, P., \& Smoot, G. 2003, A\&A, 397, 213

Paladini, R., Davies, R. D., \& De Zotti, G. 2004, MNRAS, 347, 237

Palenzuela, C., Lehner, L., \& Liebling, S. L. 2010, Sci, 329, 927

Pandey, M., Rao, A. P., Ishwara-Chandra, C. H., Durouchoux, P., \& Manchanda, R. K. 2007, A\&A, 463, 567

Pandey, M. D., Rao, A. P., Manchanda, R. K., Durouchoux, P., Ishwara-Chandra, C. H., \& Kulkarni, V. K. 2005, BASI, 33, 382

Pandey, M., Rao, A. P., Pooley, G. G., Durouchoux, P., Manchanda, R. K., \& Ishwara-Chandra, C. H. 2006, A\&A, 447, 525

Parker, E. N. 1955, ApJ, 122, 293

Patterson, C. D., Ellingson, S. W., Martin, B. S., Deshpande, K., Simonetti, J. H., Kavic, M., \& Cutchin, S. E. 2009, ACM Trans. Reconfigurable Technol. Syst., 1, 20

Patzold, M., Bird, M. K., Volland, H., Levy, G. S., Seidel, B. L., \& Stelzried, C. T. 1987, SoPh, 109, 91

Petrosian, V. 2001, ApJ, 557, 560

Petrovic, N., \& Oh, S. P. 2011, MNRAS, 413, 2103

Pfrommer, C., Enßlin, T. A., \& Springel, V. 2008, MNRAS, 385, 1211

Pfrommer, C., Springel, V., Enßlin, T. A., \& Jubelgas, M. 2006, MNRAS, 367, 113

Pindor, B., Wyithe, J. S. B., Mitchell, D. A., Ord, S. M., Wayth, R. B., \& Greenhill, L. J. 2011, PASA, 28, 46

Popov, S. B., Turolla, R., \& Possenti, A. 2006, MNRAS, 369, L23

Readhead, A. C. S. 1994, ApJ, 426, 51

Reich, W., Reich, P., \& Fuerst, E. 1990, A\&AS, 83, 539

Remillard, R. A., \& McClintock, J. E. 2006, ARA\&A, 44, 49

Rickett, B. J. 1969, Nature, 221, 158

Rickett, B. J. 1990, ARA\&A, 28, 561

Robinson, P. A., \& Cairns, I. H. 2000, in Radio Astronomy at Long Wavelengths, ed. R. G. Stone, K. W. Weiler, M. L. Goldstein, \& J.-L. Bougerot (Geophysical Monograph Series, Vol. 119; Washington, DC: American Geophysical Union), 37

Rogers, A. E. E., \& Bowman, J. D. 2008, AJ, 136, 641

Rudnick, L., et al. 2009, in Astro2010: The Astronomy and Astrophysics Decadal Survey, Science White Papers, no. 253, arXiv: 0903.0824

Ryu, D., Kang, H., Hallman, E., \& Jones, T. W. 2003, ApJ, 593, 599

Saar, S. H., \& Linsky, J. L. 1985, ApJ, 299, L47

Sagiv, A., \& Waxman, E. 2002, ApJ, 574, 861

Salah, J. E., Lonsdale, C. J., Oberoi, D., Cappallo, R. J., \& Kasper, J. C. 2005, SPIE Conf. Seri., 5901, 124
Santos, M. G., Cooray, A., \& Knox, L. 2005, ApJ, 625, 575

Scherer, K., Fichtner, H., Heber, B., \& Mall, U. 2005, Space Weather: The Physics Behind a Slogan, ed. K. Scherer, H. Fichtner, B. Heber, \& U. Mall (Berlin: Springer)

Schnitzeler, D. H. F. M., Katgert, P., \& de Bruyn, A. G. 2009, A\&A, 494, 611

Scott, D., \& Rees, M. J. 1990, MNRAS, 247, 510

Scott, S. L., Rickett, B. J., \& Armstrong, J. W. 1983, A\&A, 123, 191

Shkolnik, E., Walker, G. A. H., \& Bohlender, D. A. 2003, ApJ, 597, 1092

Skillman, S. W., Hallman, E. J., O'Shea, B. W., Burns, J. O., Smith, B. D., \& Turk, M. J. 2011, ApJ, 735, 96

Soderberg, A. M., et al. 2007, ApJ, 661, 982

Soderberg, A. M., et al. 2010, Nature, 463, 513

Sokoloff, D. D., Bykov, A. A., Shukurov, A., Berkhuijsen, E. M., Beck, R., \& Poezd, A. D. 1998, MNRAS, 299, 189

Spangler, S. R., \& Whiting, C. A. 2009, IAUS, 257, 529

Stelzried, C. T., Levy, G. S., Sato, T., Rusch, W. V. T., Ohlson, J. E., Schatten, K. H., \& Wilcox, J. M. 1970, SoPh, 14, 440

Subrahmanyan, R., \& Ekers, R. D. 2002, arXiv Astrophysics eprints, arXiv:astro-ph/0209569

Sun, X. H., Reich, W., Waelkens, A., \& Enßlin, T. A. 2008, A\&A, 477,573

Suzuki, S., \& Dulk, G. A. 1985, Bursts of Type III and Type V, ed. D. J. McLean \& N. R. Labrum (Cambridge and New York, Cambridge University Press), 289

Tammann, G. A., Loeffler, W., \& Schroeder, A. 1994, ApJS, 92, 487

Taylor, J. H., \& Cordes, J. M. 1993, ApJ, 411, 674

Taylor, A. R., Stil, J. M., \& Sunstrum, C. 2009, ApJ, 702, 1230

Tegmark, M. 1997, PhRvD, 55, 5895

Thompson, C., \& Duncan, R. C. 1995, MNRAS, 275, 255

Tingay, S. J., et al. 2013, PASA, 30

Tokumaru, M., Kojima, M., Fujiki, K., Maruyama, K., Maruyama, Y., Ito, H., \& Iju, T. 2011, Radio Sci., 46, 0

Tomsick, J. A., Corbel, S., Fender, R., Miller, J. M., Orosz, J. A., Tzioumis, T., Wijnands, R., \& Kaaret, P. 2003, ApJ, 582, 933

Usov, V. V., \& Katz, J. I. 2000, A\&A, 364, 655

Uyaniker, B., Landecker, T. L., Gray, A. D., \& Kothes, R. 2003, ApJ, 585, 785

Vršnak, B., \& Cliver, E. W. 2008, SoPh, 253, 215

Warmuth, A., \& Mann, G. 2004, LNP, 656, 49

Wieringa, M. H., de Bruyn, A. G., Jansen, D., Brouw, W. N., \& Katgert, P. 1993, A\&A, 268, 215

Wild, J. P., Smerd, S. F., \& Weiss, A. A. 1963, ARA\&A, 1, 291

Wilms, J., Pottschmidt, K., Pooley, G. G., Markoff, S., Nowak, M. A., Kreykenbohm, I., \& Rothschild, R. E. 2007, ApJ, 663, L97

Windhorst, R. A., Miley, G. K., Owen, F. N., Kron, R. G., \& Koo, D. C. 1985, ApJ, 289, 494

Wolleben, M., Landecker, T. L., Reich, W., \& Wielebinski, R. 2006, A\&A, 448, 411

Woods, P. M., \& Thompson, C. 2006, in Compact Stellar X-ray Sources, ed. W. Lewin \& M. van der Klis (Cambridge, UK: Cambridge University Press), 547

Wouthuysen, S. A. 1952, AJ, 57, 31

Wu, C. S., \& Lee, L. C. 1979, ApJ, 230, 621 
Wyithe, J. S. B. 2008, MNRAS, 387, 469

Wyithe, J. S. B., \& Loeb, A. 2008, MNRAS, 383, 606

Wyithe, J. S. B., Loeb, A., \& Barnes, D. G. 2005, ApJ, 634, 715

Wyithe, J. S. B., Loeb, A., \& Schmidt, B. P. 2007, MNRAS, 380, 1087

Wyithe, J. S. B., \& Morales, M. F. 2007, MNRAS, 379, 1647
Xiong, M., Breen, A. R., Bisi, M. M., Owens, M. J., Fallows, R. A., Dorrian, G. D., Davies, J. A., \& Thomasson, P. 2011, J. Atmos. Sol.-Terr. Phys., 73, 1270

Zarka, P., Treumann, R. A., Ryabov, B. P., \& Ryabov, V. B. 2001, Ap\&SS, 277, 293

Zaroubi, S., \& Silk, J. 2005, MNRAS, 360, L64

Zhang, X. 2007, ChJAA, 7, 712 